\title{
"Los Recursos no Renovables de las Provincias Argentinas: Consecuencias sobre el comportamiento Fiscal"
}

\begin{abstract}
$^{1}$
Este documento analiza las finanzas subnacionales en Argentina. En particular, los efectos de los ingresos por regalías sobre los recursos no renovables de las provincias, y sus posibles efectos sobre el comportamiento fiscal de estos gobiernos. En primer lugar, se describen las principales líneas de investigación relacionadas a los recursos no renovables. Después se analizan las características económicas e implicancias fiscales de estos recursos; en particular, los efectos en términos del federalismo fiscal. Seguidamente, se expone los aspectos conceptuales referidos al comportamiento fiscal de los gobiernos.

Luego, se presentan las principales estadísticas respecto a la significatividad económica y fiscal de las regalías en Latino América y particularmente en Argentina. Por último, se expone el modelo econométrico, a partir del cual se evalúa el impacto de estos recursos sobre el comportamiento fiscal subnacional.
\end{abstract}

Clasificación JEL: H71, H77, H79

Palabras Clave: Regalías, Recursos no Renovables, Ingresos y Gastos Sub nacionales, Public Choice, Federalismo Fiscal, Argentina.

Tesista: Nicolás Gonzalo Rodríguez

Director: Horacio L.P. Piffano

\footnotetext{
1 Se agradecen los valiosos aportes y comentarios realizados por el Dr. Juan Ignacio Zoloa (UNLP). Se deja constancia que lo expuesto en el presente documento es exclusiva responsabilidad del autor Nicolás Gonzalo Rodriguez.
} 
Maestría de Finanzas Públicas Provinciales y Municipales. UNLP

Facultad de Ciencias Económicas

Universidad Nacional de La Plata

Año 2017

ÍNDICE 


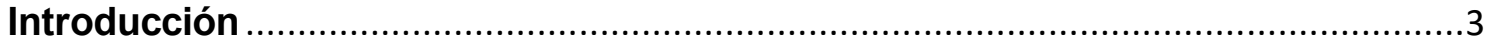

Líneas de investigación y objetivo del trabajo ....................................................

Los Recursos no Renovables (RNR): Características económicas e implicancias fiscales

La propiedad de los RNR y los regímenes e instrumentos fiscales ..........................9

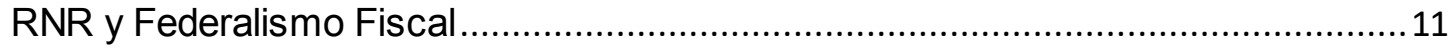

Las regalías y el comportamiento fiscal de las provincias ...................................... 14

La significatividad económica de los Recursos No Renovables (RNR) ................. 15

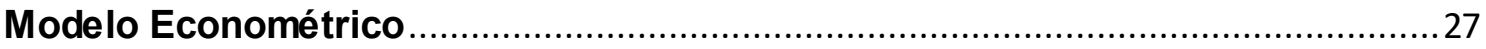

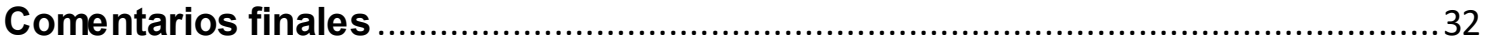

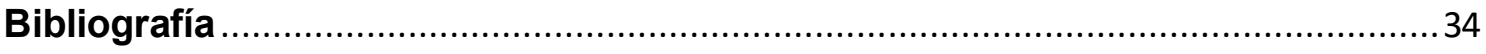

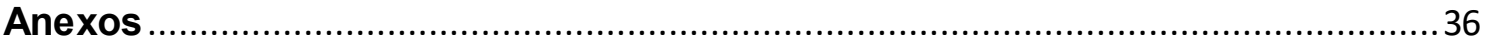

Introducción

En los últimos años, fundamentalmente a partir de principios del nuevo siglo, se ha producido una bonanza internacional en la región de América Latina, como consecuencia del aumento sostenido de los precios de los productos primarios. 
El incremento de los precios internacionales de los metales, el crudo y del resto de bienes primarios estuvo determinado, entre otros factores, por el sostenido incremento de la demanda mundial de estos bienes, explicado básicamente por el sustancial crecimiento económico de los países asiáticos en esta década. En particular, China, India, y otros países emergentes (CEPAL-UNASUR, 2013).

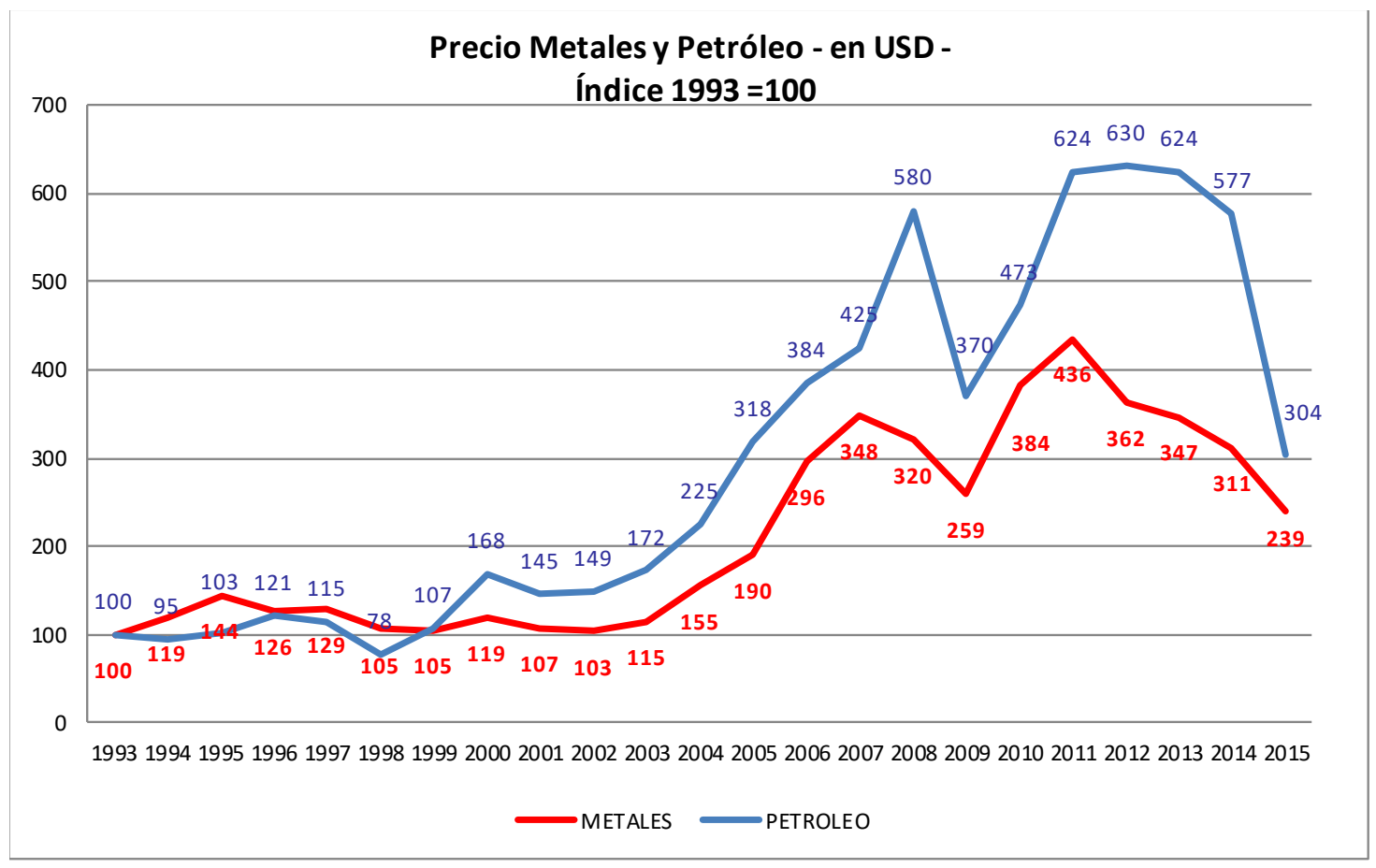

Fuente: FMI

En la actualidad, los precios de hidrocarburos y minerales se mantienen en niveles superiores en comparación con los registrados a los años previos del ciclo mencionado. El hecho de que los precios de los metales y el crudo hayan mostrado una rápida recuperación, tras su baja durante la crisis de las hipotecas de 2008 y 2009, muestra la persistencia del proceso de auge de los precios internacionales de estos bienes en este período. No obstante, como se visualiza en el gráfico previo, se registra en los últimos años una reducción en los precios de los minerales; y una reducción importante en los precios de los hidrocarburos, en particular desde el segundo semestre del $2014^{2}$.

En base a esta tendencia de la última década, "El debate sobre la dotación y el aprovechamiento sostenible y más equitativo de los recursos naturales es una prioridad de la agenda de las políticas públicas de los países de América Latina en general y de los países miembros de la Unión de Naciones Suramericanas (UNASUR) en particular" (Altomonte, CEPAL-UNASUR, 2013:7).

${ }^{2}$ La reducción en los precios, especialmente del petróleo, registrada desde 2014, no invalidan, aunque moderan las consideraciones vertidas. 
En otros términos, tal como indica Gómez Sabaíni (2015), las industrias extractivas de Recursos No Renovables (RNR) tienen considerable peso en las economías de la región, lo cual ha llevado a los distintos países, tanto por sus efectos macroeconómicos como fiscales, a implementar regímenes fiscales con el objetivo de lograr una adecuada apropiación estatal de las rentas de este sector y un adecuado manejo de sus efectos macroeconómicos. La abundancia de los mismos, su potencial exploratorio, y los elevados precios de los últimos años, determinan su papel preponderante en la economía de estos países.

Adicionalmente, como sucede en el mundo en general, una característica saliente de América Latina es la gran concentración geográfica en la disponibilidad y producción de RNR, lo cual puede generar potencialmente fuertes desequilibrios e inequidades horizontales, si estos recursos son asignados a los gobiernos subnacionales (Brosio y Giménez, 2012). Esto es lo que sucede precisamente en la Argentina, donde las provincias detentan el dominio originario sobre los RNR; y su producción, está fuertemente concentrada en algunas de las mismas.

Además de las cuestiones de equidad mencionadas, la asignación de estos recursos a las provincias, tal como se desarrollará en el presente documento, puede tener efectos no deseados sobre el comportamiento fiscal de las mismas.

En este marco, el objeto del presente documento consiste, además de dar un panorama de la especificidad económica de estos recursos y de su relevancia en la economía y finanzas públicas, en analizar los posibles efectos sobre el comportamiento fiscal que ha tenido la disponibilidad de RNR en las provincias argentinas; particularmente, en los últimos años. Las conclusiones alcanzadas pueden brindar elementos adicionales de juicio sobre los costos y beneficios de la asignación de este tipo de recursos a los gobiernos subnacionales, en un contexto de discusión sobre el federalismo fiscal en este país.

\section{Líneas de investigación y objetivo del trabajo}

La significatividad que las industrias extractivas de Recursos No Renovables (RNR) tienen en las economías de la región, motiva un mayor interés en el estudio de sus implicancias fiscales, económicas y socio ambientales. En efecto, existen distintas líneas de investigación y estudios relacionados con los RNR, y su impacto fiscal.

Uno de los principales aspectos a considerar es el uso racional de los recursos no renovables. Esto implica analizar objetivos e instrumentos de política óptima, atendiendo a las características distintivas de este tipo de recursos. En este sentido, debe tenerse presente el carácter no renovable de los mismos; esto es, lo que se extrae en un momento, deja de estar disponible para el futuro, con el consecuente costo de oportunidad. Así, resulta necesario analizar la dinámica temporal óptima en la explotación de estos recursos. 
Una línea importante de investigación se origina si se abandona el supuesto de gobierno único y se analizan los desarrollos de la teoría del federalismo fiscal con respecto a la asignación de roles y potestades de los niveles nacional y provinciales de gobierno en un sistema federal; en materia de regulación, explotación y potestad realística de los gobiernos con respecto al RNR. En esta línea, Piffano (2004) subraya que la explotación de los recursos no renovables en un sistema federal de gobierno plantea un conjunto complejo de aspectos a considerar, con vistas al diseño de la política fiscal óptima en general y de la asignación de potestades a los niveles de gobierno en un sistema federal en particular.

Un área de investigación adicional consiste en el análisis de los efectos macroeconómicos relacionados con la amplia disponibilidad de los RNR, al que suele referirse como "enfermedad holandesa". Vale recordar que, durante los años 70 , como consecuencia del descubrimiento de los campos de Groningen, hubo un importante incremento en la exportación de gas natural en los Países Bajos. Esto generó una apreciación del tipo de cambio y un aumento de las remuneraciones promedio de la economía perjudicando la industria doméstica preexistente, en particular la agricultura y las manufacturas. Este ejemplo grafica el posible impacto que tienen los RNR en la estructura productiva de una economía. Sin embargo, han aparecido enfoques que relativizan esta supuesta "maldición de los RNR"; en particular, enfatizan el rol de las instituciones en los países ricos en RNR. En este sentido, no habría problemas de "maldición" sino de mala gobernanza (Altamonte y Sánchez, 2016) ${ }^{3}$. En la misma línea, Gervasoni (2016) apunta que la condición o maldición o bendición de estos recursos dependerá de cómo se administran las rentas generadas por las mismas.

Por último, otros estudios se concentran en los aspectos ambientales vinculados a la explotación de estos recursos, y los distintos instrumentos de política pública (regulatorios, fiscales) disponibles para lograr una explotación sustentable desde esta perspectiva.

Teniendo en cuenta estas consideraciones, la motivación de este trabajo consiste en analizar el efecto que tiene la disponibilidad de los RNR en el comportamiento fiscal de las provincias, en línea con la segunda línea de investigación mencionada. En particular, se pretende determinar si la disponibilidad de recursos fiscales provenientes de la explotación de los Recursos no Renovables (específicamente hidrocarburos y minería), ha determinado una reducción o "relajamiento" en el "Esfuerzo Fiscal" de las provincias a la hora de recaudar el resto de los tributos subnacionales; y/o un incentivo a incrementar más allá de lo óptimo el gasto público y la cantidad de empleados provinciales de las jurisdicciones. Así, por ejemplo, pueden registrarse, según aspectos conceptuales del federalismo fiscal, efectos sobre los incentivos a un menor esfuerzo en recaudar impuestos subnacionales por parte de las mismas, dado el

3 Buena gobernanza implica Instrumentar fondos de estabilización, reglas macro fiscales, fondos de ahorro e inversión, lo cual evitaría consumir en el corto plazo (como sucede en general en América Latina) las rentas extraordinarias de estos recursos en períodos de holgura. También respeto de derecho de propiedad, y marcos fiscales y regulatorios adecuados para el sector. 
mayor costo político de recaudar estos últimos, en comparación con los provenientes de las regalías; además de generar disparidades significativas en el financiamiento de las provincias, entre otros efectos significativos. En otro sentido, puede generarse incentivos a sobre expandir el gasto público de las provincias, al no internalizarse en los policy-makers apropiadamente los costos económicos y políticos de este tipo de recursos fiscales.

En el primer apartado del trabajo, se presentan las principales características económicas de los RNR y su impacto fiscal. Seguidamente, se analiza los distintos esquemas de propiedad de los RNR, y los instrumentos fiscales específicos aplicados sobre el sector. A continuación, se presenta los aspectos generales vinculados a los RNR y el Federalismo Fiscal; y en particular, las posibles consecuencias sobre el comportamiento fiscal de los policy-makers de las jurisdicciones subnacionales. Luego, el documento se concentra en el análisis descriptivo y estadístico del sector vinculado a los RNR, tanto para América Latina como para la Argentina en particular. Seguidamente, se encara el análisis econométrico, a fin de evaluar el impacto de los ingresos por RNR sobre los principales agregados fiscales de las provincias. Específicamente, se analiza la relación entre el esfuerzo fiscal de las provincias en función de la disponibilidad de recursos fiscales provenientes de RNR, como así también el efecto de estos últimos recursos sobre el gasto de las provincias y la dotación de empleados públicos de las mismas. Finalmente, se presentan las principales conclusiones y comentarios finales.

\section{Los Recursos no Renovables (RNR): Características económicas e implicancias fiscales}

Gómez Sabaíni (2015) apunta que las industrias extractivas de recursos naturales no renovables, a diferencia de las actividades productivas tradicionales, están sujetas a un proceso variable, pero a la vez inexorable de agotamiento físico. En tal sentido, resulta claro el costo de oportunidad que posee la decisión de extraer recursos hoy en detrimento de su disponibilidad en el futuro.

Siguiendo los lineamientos conceptuales de Piffano (2004), suponiendo la existencia de propiedad pública del recurso (supuesto que será levantado en aras del presente trabajo), el primer problema que enfrenta el gobierno es el de la distribución intergeneracional del uso del recurso. Así, resulta necesario prever un cierto sendero de su explotación y la constitución de reservas o fondos de ahorro, con la finalidad de garantizar niveles estables de consumo a las futuras generaciones ${ }^{4}$. En segundo término, se debe atender a la distribución intertemporal de su uso ante escenarios inciertos en materia de ingresos futuros y el diseño de esquemas de ahorro precautorio, debido a modificaciones en el nivel de explotación y en el precio relativo del recurso. Relacionado con este punto, se plantea asimismo la cuestión de la sustentabilidad

\footnotetext{
${ }_{4}$ En este punto, deben tenerse presente consideraciones de largo plazo. Por ejemplo, la posibilidad de una caída en el precio del petróleo ante la sustitución de los automotores a combustión interna por energías más limpias (sin ir más lejos; algunas organizaciones europeas (Climate Action Tracker) pronostican los últimos automóviles producidos con dicha energía en 2035).
} 
fiscal de mediano y largo plazo de las finanzas del gobierno. En tercer lugar, en los casos en que la participación de la renta del recurso (regalías) sea significativa como fuente de financiamiento de los gobiernos, los cambios en los precios de mercado del recurso plantean la existencia de costos de transacción, en términos de los denominados costos de los ajustes presupuestarios. Por último, el autor establece que la forma de instrumentar los ahorros presentes, en aras de salvaguardar objetivos de política intergeneracionales y de ahorro precautorio, conduce al análisis de instrumentos financieros alternativos, en particular los ligados al diseño y operación de los fondos de estabilización y/o fondos de ahorro.

Por su parte, Altamira y Sánchez (2016) resaltan la necesidad de destinar las rentas extraordinarias de estos recursos hacia inversiones de largo plazo. Tal como mencionan en su trabajo: "los usos tradicionales de estas rentas orientadas al corto plazo resultan contradictorio con la propia naturaleza en que se basan los recursos de las actividades extractivas, ya que se trata por definición de recursos no renovables". ${ }^{5}$

Por otro lado, Gómez Sabaíni (2015) plantea que la explotación económica de hidrocarburos y minerales poseen ciertas características específicas que deben ser mencionadas: las grandes inversiones al inicio de los proyectos (costos hundidos) que requieren de un largo tiempo de producción continua para ser recuperados; las sustanciales rentas económicas que generan y los ingresos fiscales que pueden obtenerse a partir de las mismas; la elevada incertidumbre que rodea al sector (volatilidad del precio internacional y nivel potencial de producción sujeto a hallazgos geológicos, costo de los insumos); las asimetrías de información en cuanto a la estructura de costos y aspectos técnicos de la exploración, desarrollo y producción en favor del inversor productor, $\mathrm{y}$ en cuanto a las intenciones y decisiones políticas futuras en favor del gobierno; la multiplicidad de negocios adicionales que pueden desarrollarse en torno a la explotación básica; el considerable poder de mercado que pueden tener determinados productores cuando poseen una porción significativa de las reservas mundiales y un nivel de producción en consonancia de alguno de estos recursos no renovables.

El carácter de recurso no renovable, juntamente con las características intrínsecas del sector mencionadas, tienen implicancias económicas y fiscales distintivas. Concretamente, la incidencia económica de las regalías sobre los RNR tiene características distintivas al tradicional de un impuesto a la producción. Así, un impuesto a la explotación de un recurso "reproducible" genera carga excedente debido al "excedente perdido" (o dejado de obtener) por el productor y/o consumidor. Esto resulta diferente en el caso de la explotación de un recurso "agotable". En este sentido, si el gobierno limita su explotación en determinado año, por ejemplo, ello no impide que al año siguiente se lo haga, sin perder, en términos Inter

\footnotetext{
${ }^{5}$ Melamud y Rozenwursen (2016), van más allá y afirman que la disponibilidad de estos recursos, al utilizarlas en inversiones de largo plazo, puede aliviar la escasez de capital en los países subdesarrollados e impulsar su desarrollo, aún en actividades no relacionadas con los RNR. Sin embargo, el carácter fungible del dinero puede hacer que afectar estos recursos a gastos de capital tenga un resultado relativo.
} 
temporales, los niveles de producción. La decisión de hacerlo hoy o mañana en realidad depende de cómo se ponderen las funciones de utilidad de la presente y futura generación, pues el recurso es agotable ("no reproducible") ${ }^{6}$.

\section{La propiedad de los RNR y los regímenes e instrumentos fiscales}

Generalmente, al menos en los países de América Latina y el Caribe, suele reconocerse que los acervos de minerales e hidrocarburos que yacen en el subsuelo, así como el resto de recursos naturales, pertenecen a los Estados soberanos e integran el dominio público de sus ciudadanos.

En la Argentina, el artículo 124 de la Constitución Nacional dispuso: "Corresponde a las provincias el dominio originario de los recursos naturales existentes en su territorio". A partir de esta disposición, queda establecido que los RNR quedan en cabeza de las provincias.

Sin perjuicio de lo anterior, el Estado nacional tiene ciertas potestades sobre el sector a ser consideradas. En primer lugar, detenta un dominio residual sobre los RNR. En segundo lugar, tiene la capacidad de aplicar tributos de carácter nacional generales o específicos sobre el sector; y establece los derechos de importación y exportación. También, se reserva su carácter de regulador de la política nacional sobre los RNR, amparado en el artículo 75 de inc.18 de la Constitución Nacional. Especificamente, tal como menciona Brosio y Jiménez (2012) puede regular los precios internos, lo cual fue particularmente importante en la década anterior.

Una vez reconocido el dominio sobre los RNR, resulta esencial diseñar un régimen fiscal específico para asegurar la apropiación estatal de una razonable porción de las rentas generadas en las respectivas industrias extractivas.

De esta forma, el diseño de un régimen fiscal sobre recursos no renovables suele plantear una serie de desafíos en cuanto a los objetivos no siempre alineados entre sí. El principal de ellos consiste en lograr un balance entre el eventual y/o generalmente intento del gobierno de maximizar los ingresos fiscales derivados de estos sectores y los incentivos que se ofrecen a los agentes privados para garantizar su participación en las actividades de su exploración y en la producción de los derivados. Una presión fiscal muy elevada sobre este sector puede atentar contra los incentivos a invertir por parte de las empresas privadas, al reducir los márgenes de ganancia; y así reducir eventualmente la recaudación potencial de los gobiernos, contrariamente al objetivo buscado. Tal como se destaca en el documento realizado por el FMI (2012), el principal "issue" fiscal consiste en asegurar una participación "razonable" en las rentas capturadas por los gobiernos. Se entiende por "razonable" aquella participación que como mínimo deja a los inversores privados adecuados incentivos a explorar, desarrollar y

${ }^{6}$ Por cierto, el ritmo de explotación también depende de la expectativa que se tenga respecto a la evolución futura del precio de los RNR, las expectativas sobre cambio tecnológico, las reservas comprobadas o a descubrir, etc. (Piffano, 2004). 
producir. También, debe tenerse presente, para la instrumentación de los distintos regímenes fiscales, atender a las características distintivas de los sectores de hidrocarburos y de minería respectivamente (FMI, 2012). En el sector de hidrocarburos, por ejemplo, los costos y riegos involucrados en la etapa de exploración son sustancialmente mayores. Sin embargo, en las etapas de desarrollo y extracción, los riesgos pueden ser mayores en la minería; además de los riesgos políticos y de medio ambiente involucrados en este último.

En las industrias extractivas existe básicamente dos tipos de regímenes fiscales: los sistemas de concesiones y los sistemas contractuales (Gómez Sabaíni, 2015).

En los acuerdos de concesión generalmente el Estado otorga a una compañía el derecho exclusivo a explorar, desarrollar, producir, transportar y comercializar los recursos naturales extraídos asumiendo todos los riesgos y costos asociados a la explotación dentro de un área delimitada y por un periodo de tiempo determinado. En general, los operadores privados son los propietarios legales de la producción, no así los recursos que permanecen en el terreno adjudicado, y tiene libertad para disponer de la misma. Por su parte, el Estado sólo participa en la generación de rentas económicas, básicamente a través del cobro de regalías y o impuestos.

Con el tiempo, los sistemas de concesiones comenzaron a ser vistos como incompatibles con la soberanía estatal. De hecho, los sistemas contractuales surgieron como resultado de los esfuerzos para modificar la naturaleza de las relaciones entre las grandes empresas privadas internacionales y los países con disponibilidad de recursos no renovables. Bajo un sistema contractual típico el Estado es el propietario no sólo del recurso natural sino de la producción que obtiene la empresa privada, la cual opera a su propio riesgo y costos bajo el control estatal y recibe un pago en especie o en efectivo como contrapartida si la producción resulta exitosa.

En la práctica internacional suelen encontrarse ejemplos de ambos sistemas aplicados a la producción de petróleo y gas, no así en el caso de la actividad minera donde la explotación de los recursos generalmente se realiza a través de concesiones a empresas privadas (Gómez Sabaíni, 2015).

Históricamente los instrumentos más comúnmente utilizados tanto en el sector de hidrocarburos como en la minería han sido las regalías, ya sea que están definidas por unidad producida (adquantum) o a través de una variable económica ad valorem; y el tradicional impuesto sobre la renta societaria.

Adicionalmente en varios países, como en la Argentina, los regímenes fiscales sobre las industrias extractivas se completan mediante la aplicación de impuestos que generalmente integran el régimen general de tributación como el IVA, algunos impuestos selectivos, o los gravámenes sobre el comercio internacional, tanto sobre las exportaciones como sobre las importaciones. 


\section{RNR y Federalismo Fiscal}

Los estudios sobre Federalismo Fiscal tienen como objeto principal analizar la asignación de potestades de recaudación y responsabilidades de gasto entre los distintos niveles de gobierno (nivel nacional y subnacional).

Uno de los puntos centrales consiste en analizar el grado óptimo de descentralización fiscal. Para ello, se contrastan los beneficios y costos involucrados en descentralizar (o centralizar) las potestades y responsabilidades fiscales entre el gobierno central y los gobiernos subnacionales. La evaluación de esta temática resulta por demás compleja ya que existen múltiples variables a considerar (no solo económicas, sino de administración, de economía política, etc.), y siempre existen ventajas y desventajas en torno a cada alternativa. Sin embargo, existe cierto consenso sobre las virtudes de la descentralización, y una tendencia en la práctica hacia la descentralización fiscal a los gobiernos subnacionales. Un marco analítico básico para analizar esta cuestión y justificar el proceso de descentralización está dado por el teorema de descentralización de Oates.

El teorema de la descentralización de Oates sostiene que, en ausencia de ahorro de costos por la provisión centralizada (a nivel nacional) de los bienes públicos, el bienestar será siempre mayor si se proveen de manera descentralizada por las jurisdicciones (provincias). Esto se debe a que la descentralización permite adaptar la oferta de bienes públicos a la demanda de los ciudadanos de cada jurisdicción. Los votantes a quienes no les gusta la oferta de bienes públicos tienen al menos la opción de marcharse a otra jurisdicción, es decir, "votar con los pies" (Teorema de Tiebout). Así, desde esta perspectiva, se logra un mayor bienestar social, al respetar mayormente las preferencias individuales de los ciudadanos con relación a la provisión de bienes públicos.

Específicamente Oates (1972), concluye que un sistema descentralizado ofrece:

1) Posibilidades de mayor eficiencia económica, al proporcionar niveles de servicios más adecuados a las preferencias de los distintos grupos de consumidores, hecho que se refuerza con la movilidad de los consumidores (Tiebout 1956).

2) Mayores posibilidades de progreso técnico en la producción de estos bienes, por mayor experimentación, innovación y emulación entre productores.

3) Mayor eficiencia en el gasto, ya que la prestación de los servicios estará mucho más próxima a sus costes reales si la comunidad se ve obligada a financiar su propio programa público a través de la tributación local.

Por otra parte, los enfoques de federalismo fiscal de "segunda generación" hacen hincapié en las bondades de la descentralización a partir de un mayor control sobre el comportamiento de 
los gobernantes. Tal como afirma Tanzi (2016), la escuela del "public choice" estableció la idea de que la descentralización es una manera de restringir el poder del "Estado Leviatán" en su objetivo de expandir su poder sobre la sociedad. Así, un mayor nivel de descentralización puede mejorar el comportamiento fiscal de los policy-makers al acercar la toma de decisiones a los ciudadanos, permitiendo un mayor control por parte de los mismos. También mejoraría la participación democrática: "La literatura sugiere que cuanto mayor partición (estructura multinivel, con unidades de gobierno pequeñas) y mayores responsabilidades asignadas a los gobiernos locales democráticos, mayor será la participación política” (Porto, 2004:42).

En términos generales, se menciona tres méritos de la descentralización: eficiencia en la asignación de recursos, mayor responsabilidad fiscal y rendición de cuentas, y mejor matching con las preferencias de los ciudadanos (Tanzi, 2016).

Sin embargo, la descentralización implica costos, con lo cual la decisión surgirá de la comparación entre estos últimos y los beneficios mencionados en los párrafos precedentes (trade-offs). Por ejemplo, los gobiernos locales pueden incurrir en impuestos distorsivos, por ejemplo, por tener que establecer impuestos sobre bases móviles; y también, suelen existir economías de escala en la recaudación al centralizar la misma en el gobierno central. Asimismo, la descentralización fiscal deja un menor margen para realizar transferencias que aseguren la equidad regional entre las provincias rezagadas y las de mayor desarrollo relativo.

Otro de los problemas vinculados a la descentralización, se relaciona con la posibilidad de descentralizar los gastos sin la correspondiente descentralización de la responsabilidad de recaudar los recursos fiscales, originando la falta de la denominada correspondencia fiscal. En este sentido, los aportes teóricos de la escuela del "public choice" enfatizan la necesidad de descentralizar simultáneamente, los gastos y los recursos. "EI punto tiene que ver con los efectos provocados por la separación de las decisiones de gasto y su financiamiento" (Piffano, Nota 1, 2005:39); este enfoque predice que esta separación puede inducir a los gobernantes que deciden el gasto, pero no tienen la responsabilidad total de su financiamiento (parte del mismo proviene de transferencias del gobierno central) a tener un comportamiento menos responsable sobre el gasto que si se hiciera cargo del total financiamiento. Tal como cita Tanzi (2016:31), "the claims that descentralization enhances service delivery fail to recognize the joint nature of spending and revenue contraints".

Más allá de las consideraciones generales sobre federalismo fiscal, en el caso específico de los RNR, la mayoría de los argumentos favorecen la centralización de los RNR en el gobierno central; en tanto los argumentos en favor de la descentralización son de carácter fundamentalmente histórico, político e institucional, tal como sucede en la Argentina.

De acuerdo con el enfoque normativo del federalismo fiscal, las ventajas a favor de la centralización de la explotación de los recursos naturales no renovables están ligados a 
aspectos de eficiencia económica y correspondencia fiscal (accountability), estabilización macroeconómica y equidad distributiva (Piffano, 2004).

En materia de estabilización macroeconómica, los gobiernos centrales están en mejor situación que los gobiernos subnacionales para absorber la incertidumbre y volatilidad de los precios del recurso, al contar con una base tributaria mucho más amplia en gran parte no correlacionada con los precios de los RNR.

En cuanto al ámbito de la eficiencia económica y la correspondencia fiscal, los tributos subnacionales deben basarse esencialmente en bases no móviles con una importante dosis de sustento en el principio del beneficio, de forma que los electores subnacionales adviertan con nitidez el costo de oportunidad de sus demandas por bienes públicos subnacionales. Específicamente, el financiamiento con el uso de una fuente de ingresos "agotables", puede distorsionar la percepción de dicho costo de oportunidad, y generar un efecto similar al "flypaper effect" sobre el nivel del gasto de las provincias.

Por su parte, Brosio y Jiménez (2012), enfatizan consideraciones de eficiencia económica, en favor de la centralización de los RNR, vinculadas a la movilidad de los factores productivos. En esta línea, desarrollan el ejemplo de un descubrimiento de RNR, que permite (a partir de los ingresos fiscales extraordinarios) reducir la presión tributaria en el resto de los tributos. Esto, aumenta el beneficio (o residuo fiscal) de los pobladores de la jurisdicción atrayendo "artificialmente" más trabajadores de otros estados (lo cual es ineficiente desde el punto de vista de asignación de recursos).

Finalmente, en cuanto a la equidad distributiva, las asimetrías en los ingresos regionales pueden más fácilmente atenuarse (mediante transferencias de nivelación) a través del gobierno central que explote el rendimiento fiscal del recurso no renovable; un aspecto usual de equidad horizontal en sentido territorial. De esta forma, el reconocimiento de potestades tributarias a los gobiernos subnacionales debe coincidir con fuentes que resulten mayormente uniformes en su distribución espacial. Cuando las asimetrías regionales entre todas las bases potenciales de los gobiernos regionales o locales, se torna extrema - como es el caso de contar o no contar con RNR en el subsuelo - la posibilidad de nivelación regional se torna extremadamente dificultosa de perseguir.

Tal como expone Fletes Cibils (2016:59), "la concentración de estos recursos (regalías) en pocas jurisdicciones subnacionales, a menudo en una escala desproporcionada en relación con sus necesidades de gasto y rendición de cuentas puede dar lugar a gastos innecesarios y corrupción; al mismo tiempo, pueden reducir los incentivos para movilizar otras formas de ingresos propios". 


\section{Las regalías y el comportamiento fiscal de las provincias}

En este punto del trabajo, ya es posible delinear algunas de las posibles consecuencias de la disponibilidad de RNR, específicamente sobre el comportamiento y los resultados fiscales de las provincias.

Piffano (2004) explica que desde el punto de vista de los gobernantes de turno existe una gran diferencia entre el asumir el costo político de establecer tributos a los ciudadanos electores para financiar el gasto público, y la alternativa - sin tales costos políticos - de ejercer la potestad regalística como fuente de financiamiento. Las regalías constituyen un recurso no tributario, que ni siquiera surge del bolsillo de los ciudadanos de la generación presente a quienes, por lo tanto, se habrá de proveer bienes públicos "sin costos". Esto puede tender a expandir el gasto más allá de lo óptimo, al no internalizarse de forma plena el costo económico de financiar el gasto público.

Asimismo, si los gobiernos subnacionales cuentan con otros recursos tributarios asignados, las regiones ricas poseedoras de RNR tendrían muy poco incentivo a explotar esas otras bases tributarias. El efecto es conocido como "beggar-the-neighbor" (empobrecimiento del vecino) al poner en franca desventaja a los gobiernos subnacionales vecinos que no cuentan con estos recursos y deben gravar las otras bases; e inducir fiscalmente reasignaciones espaciales no eficientes de los factores de la producción.

Paralelamente, un enfoque conceptual para analizar los resultados expuestos en este trabajo proveniente de las ciencias políticas. En particular, Gervasoni (2010) refiere al concepto de "Estado Rentístico" para referirse a Estados fiscalmente ricos capaces de distribuir recursos casi sin cobrar impuestos gracias a la apropiación de enormes rentas provenientes de recursos no naturales (RNR).

"En esta situación, los Estados rentistas tienen acceso a los ingresos procedentes de fuentes independientes en gran medida de la amplia variedad de impuestos, y estos ingresos pueden ser muy grandes vis-à-vis la economía nacional. En tales condiciones, el Estado es la principal fuente de riqueza. En este último caso, podrían utilizarlos para pagar los altos salarios de empleados públicos, la adjudicación de costosas adquisiciones, financiar excesiva clientela, y dominar el mercado de publicidad en los medios de comunicación. Así, en estas provincias podría registrarse un mayor tamaño del Estado y o mayor dotación de empleados públicos" Gervasoni (2010:307).

Según el autor citado, el funcionamiento del federalismo fiscal en la Argentina es para muchas provincias una fuente de rentas. Esto es, si recibe transferencias federales muy por encima de lo que podría lograr por sus propios medios, estas transferencias equivalen a rentas provenientes de RNR. Si además esas provincias hacen muy poco esfuerzo fiscal, entonces se 
trata de Estados rentísticos. Los Estados gozan así de los beneficios de gastar sin pagar los costos políticos de recaudar.

\section{La significatividad económica de los Recursos No Renovables (RNR)}

En los siguientes dos cuadros, se presentan distintos indicadores que dan cuenta de la significatividad de los RNR (en particular, Hidrocarburos y Minería) en la economía de países de Latinoamérica; en tres periodos distintos: 2003 a 2005 (antes del inicio del ciclo alcista de precios); 2005 a 2008 (años de alto crecimiento en el precio internacional de los productos primarios); y 2010 a 2013 (después de la crisis financiera internacional).

En primer lugar, se visualiza la significatividad de los Ingresos fiscales provenientes de estos sectores económicos en el PIB de cada país ${ }^{7}$. Así, por ejemplo, se destaca la participación de los ingresos fiscales del sector Hidrocarburos en la economía de Ecuador, Bolivia y Venezuela; llegando a representar, en el último período, entre el $13 \%$ y $10 \%$ del PIB de esos países (en Argentina representa el $1 \%$ del PIB, prácticamente el más bajo de la muestra de países).

El segundo indicador mide la relación de los ingresos fiscales provenientes de estos recursos con relación a la renta económica del sector. Así, se destaca Bolivia, México y Ecuador, donde los ingresos fiscales provenientes de hidrocarburos se ubican entre el $70 \%$ y el $75 \%$ de la renta de este sector en los últimos años ( $26 \%$ en el caso de Argentina, el más bajo de la muestra).

Por último, el tercer ratio refleja la importancia de estos recursos fiscales con relación al total de ingresos fiscales del país en cuestión. En este caso, se destaca Venezuela y Ecuador, donde significan más del $40 \%$ de los ingresos fiscales totales (en Argentina sólo explican el 3,9\% del total de ingresos fiscales).

Los datos permiten observar, además de una gran dispersión entre los países, un aumento generalizado de la participación del Estado en la renta del sector Hidrocarburífero (aunque se mantiene casi constante en Brasil y Venezuela).

\footnotetext{
7 Ingresos Fiscales incluyen el total de la carga tributaria sobre el sector (no sólo regalías). En Argentina, por ejemplo, incluye Regalías (incluyendo en el cuadro a las de Minería), impuesto a la renta y Derechos de exportación (ver Galiani S. 2015). A estos impuestos podría sumarse lo recaudado en concepto de otros impuestos como impuesto sobre los Ingresos Brutos, entre otros, lo cual daría una presión tributaria mayor que la consignada (ver Einstoss Tinto A., 2016).
} 
Indicadores relativos a ingresos fiscales generados por la producción de Hidrocarburos

\begin{tabular}{|c|c|c|c|c|c|c|c|c|c|}
\hline \multirow[b]{2}{*}{ Países } & \multicolumn{3}{|c|}{$\% \mathrm{~s} / \mathrm{PBI}$} & \multicolumn{3}{|c|}{$\%$ s/ Renta del Sector } & \multicolumn{3}{|c|}{$\%$ s/ Ingr. Fiscales Totales } \\
\hline & $\begin{array}{c}2000 \text { a } \\
2003\end{array}$ & $\begin{array}{c}2005 \mathrm{a} \\
2008\end{array}$ & $\begin{array}{c}2010 \text { a } \\
2013\end{array}$ & $\begin{array}{c}2000 \mathrm{a} \\
2003\end{array}$ & $\begin{array}{c}2005 a \\
2008\end{array}$ & $\begin{array}{c}2010 \text { a } \\
2013 \\
\end{array}$ & $\begin{array}{c}2000 \mathrm{a} \\
2003\end{array}$ & $\begin{array}{c}2005 \text { a } \\
2008\end{array}$ & $\begin{array}{c}2010 a \\
2013\end{array}$ \\
\hline Argentina & $1 \%$ & $2 \%$ & $1 \%$ & $15 \%$ & $18 \%$ & $26 \%$ & $5 \%$ & $7 \%$ & $4 \%$ \\
\hline Bolivia & $3 \%$ & $9 \%$ & $10 \%$ & $28 \%$ & $26 \%$ & $74 \%$ & $11 \%$ & $28 \%$ & $30 \%$ \\
\hline Brasil & $1 \%$ & $1 \%$ & $1 \%$ & $37 \%$ & $38 \%$ & $36 \%$ & $3 \%$ & $4 \%$ & $3 \%$ \\
\hline Colombia & $2 \%$ & $2 \%$ & $3 \%$ & $28 \%$ & $27 \%$ & $39 \%$ & $5 \%$ & $7 \%$ & $12 \%$ \\
\hline Ecuador & $6 \%$ & $9 \%$ & $13 \%$ & $45 \%$ & $35 \%$ & $69 \%$ & $29 \%$ & $35 \%$ & $40 \%$ \\
\hline Mexico & $3 \%$ & $6 \%$ & $6 \%$ & $73 \%$ & $70 \%$ & $77 \%$ & $21 \%$ & $38 \%$ & $34 \%$ \\
\hline Peru & $1 \%$ & $1 \%$ & $1 \%$ & $35 \%$ & $38 \%$ & $54 \%$ & $3 \%$ & $6 \%$ & $7 \%$ \\
\hline Venezuela & $11 \%$ & $14 \%$ & $11 \%$ & $39 \%$ & $37 \%$ & $36 \%$ & $48 \%$ & $51 \%$ & $45 \%$ \\
\hline Media (menos Arg.) & $4 \%$ & $6 \%$ & $6 \%$ & $41 \%$ & $39 \%$ & $55 \%$ & $17 \%$ & $24 \%$ & $24 \%$ \\
\hline
\end{tabular}

Fuente: En base a Gomez Sabaini, 2015

En Argentina incluye Regalías, Ganancias de Sociedades y Derechos de Exportación

En el cuadro siguiente, se presentan los mismos indicadores para el sector de minería. En este caso, la participación del sector, medido por cualquiera de los indicadores, es significativamente menor que en el caso de Hidrocarburos.

Respecto al primer indicador, se destaca Chile, donde, en los últimos años, los ingresos fiscales significan el $3,4 \%$ del PIB $(0,1 \%$ en el caso de Argentina, el más bajo de la muestra).

Respecto al segundo indicador, sobresale Bolivia, donde los mismos significan casi el $24 \%$ de la renta del sector (casi el $20 \%$ en Argentina, segundo después de Chile).

Por último, Chile se destaca por la participación de estos recursos con relación a los ingresos fiscales totales, superando el $15 \%$ de los mismos en el período 2010 a1013 (0,5\% en Argentina, casi el más bajo de la muestra).

Los datos evidencian una dinámica muy heterogénea entre los países. Así, por ejemplo, los ingresos fiscales recaudados sobre RNR respecto a la renta del sector, se incrementan en países como Argentina, Brasil, Chile; en tanto se reduce en Bolivia, Colombia México y Perú.

Indicadores relativos a ingresos fiscales generados por la producción de Minería

\begin{tabular}{|c|c|c|c|c|c|c|c|c|c|}
\hline \multirow[b]{2}{*}{ Países } & \multicolumn{3}{|c|}{$\% \mathrm{~s} / \mathrm{PBI}$} & \multicolumn{3}{|c|}{$\%$ s/ Renta del Sector } & \multicolumn{3}{|c|}{$\%$ s/ Ingr. Fiscales Totales } \\
\hline & $\begin{array}{c}2000 \mathrm{a} \\
2003\end{array}$ & $\begin{array}{c}2005 \mathrm{a} \\
2008\end{array}$ & $\begin{array}{c}2010 \mathrm{a} \\
2013\end{array}$ & $\begin{array}{c}2000 \mathrm{a} \\
2003\end{array}$ & $\begin{array}{c}2005 a \\
2008\end{array}$ & $\begin{array}{c}2010 a \\
2013\end{array}$ & $\begin{array}{c}2000 \mathrm{a} \\
2003\end{array}$ & $\begin{array}{c}2005 a \\
2008\end{array}$ & $\begin{array}{c}2010 a \\
2013\end{array}$ \\
\hline Argentina & $0,0 \%$ & $0,1 \%$ & $0,1 \%$ & $2,0 \%$ & $22,4 \%$ & $19,8 \%$ & $0,0 \%$ & $0,4 \%$ & $0,5 \%$ \\
\hline Bolivia & $0,1 \%$ & $0,7 \%$ & $1,1 \%$ & $101,9 \%$ & $22,5 \%$ & $23,6 \%$ & $0,3 \%$ & $2,1 \%$ & $3,2 \%$ \\
\hline Brasil & $0,0 \%$ & $0,2 \%$ & $0,2 \%$ & $2,1 \%$ & $5,8 \%$ & $8,6 \%$ & $0,1 \%$ & $0,5 \%$ & $0,7 \%$ \\
\hline Chile & $0,8 \%$ & $6,9 \%$ & $3,4 \%$ & $12,6 \%$ & $36,6 \%$ & $20,3 \%$ & $4,0 \%$ & $27,7 \%$ & $15,3 \%$ \\
\hline Colombia & $0,2 \%$ & $0,5 \%$ & $0,4 \%$ & $58,5 \%$ & $21,6 \%$ & $15,9 \%$ & $0,6 \%$ & $1,6 \%$ & $1,3 \%$ \\
\hline Mexico & $0,0 \%$ & $0,1 \%$ & $0,2 \%$ & $71,8 \%$ & $31,3 \%$ & $16,3 \%$ & $0,3 \%$ & $0,8 \%$ & $1,0 \%$ \\
\hline Peru & $0,2 \%$ & $2,1 \%$ & $1,5 \%$ & $43,3 \%$ & $22,1 \%$ & $14,9 \%$ & $1,0 \%$ & $10,6 \%$ & $7,4 \%$ \\
\hline Media (menos Arg.) & $0,2 \%$ & $1,8 \%$ & $1,1 \%$ & $48,4 \%$ & $23,3 \%$ & $16,6 \%$ & $1,1 \%$ & $7,2 \%$ & $4,8 \%$ \\
\hline
\end{tabular}

De los datos presentados, puede concluirse que Argentina se encuentra entre los países de la muestra donde la significatividad de los recursos fiscales provenientes de RNR resulta más 
baja. Esta situación se evidencia particularmente con relación al $\mathrm{PBI}$ y los ingresos fiscales totales (primer y tercer indicador); no tanto así con relación a la renta económica del sector (sobre todo en minería). Esto sugiere un mayor potencial en la producción de RNR que en una mayor presión tributaria sobre los mismos.

\section{La Argentina:}

La República Argentina está caracterizada, como otros países de América Latina y el Caribe, por la riqueza de su suelo en Recursos No Renovables (RNR), tales como hidrocarburos (fundamentalmente petróleo y gas), como los provenientes de la minería (oro, plata, entre otros).

Amén de esta riqueza, las políticas macroeconómicas implementadas en la última década no fueron particularmente beneficiosas para el desarrollo del sector, y la consecuente recaudación fiscal. Por un lado, como consecuencia del descalce de los precios internos y externos en el sector hidrocarburifero. Por otro lado, a partir de la falta de reglas de juego claras e inseguridad jurídica, que desalentaron grandes inversiones privadas en el sector.

En el siguiente cuadro, se visualiza la significatividad de la producción de RNR en la economía. Después de alcanzar un pico del $6 \%$ en el año 2002 y 2003, su significatividad se reduce sistemáticamente, en particular a partir del año 2006.

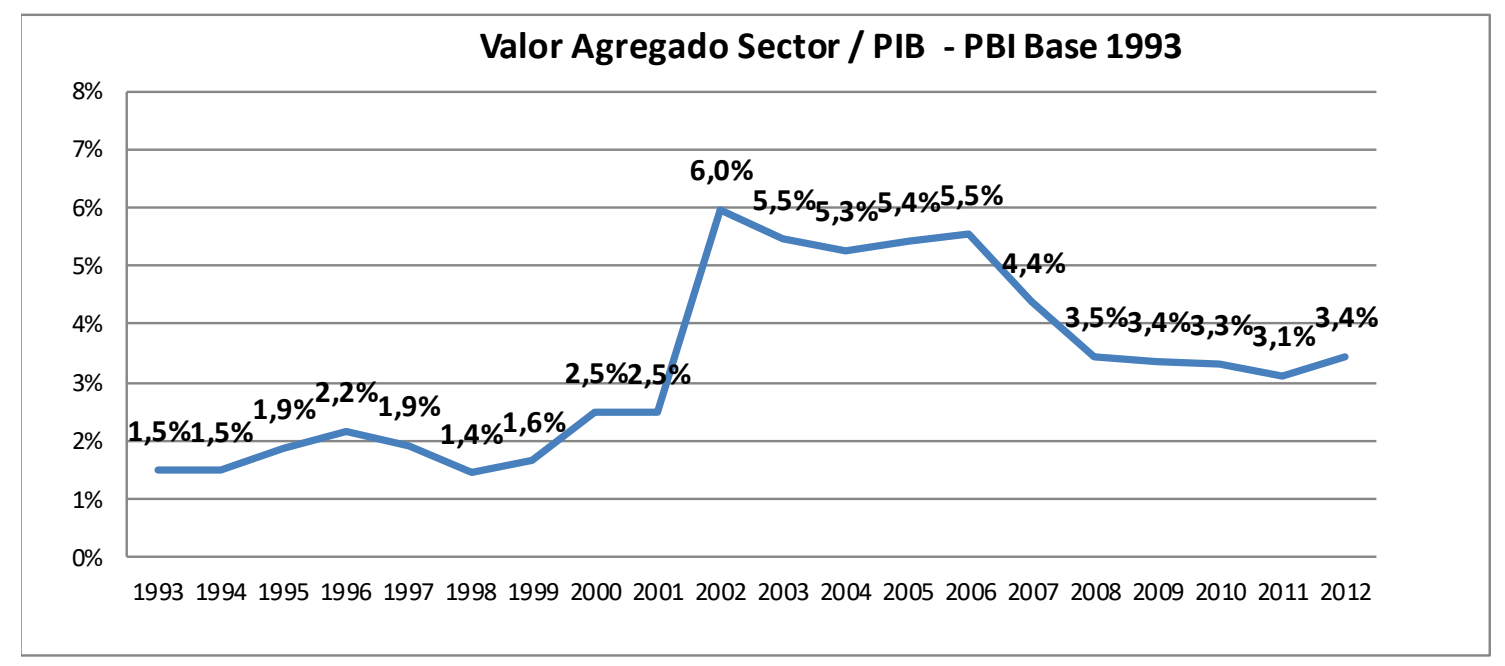

Fuente: INDEC. Sector "Explotación de minas y canteras" 


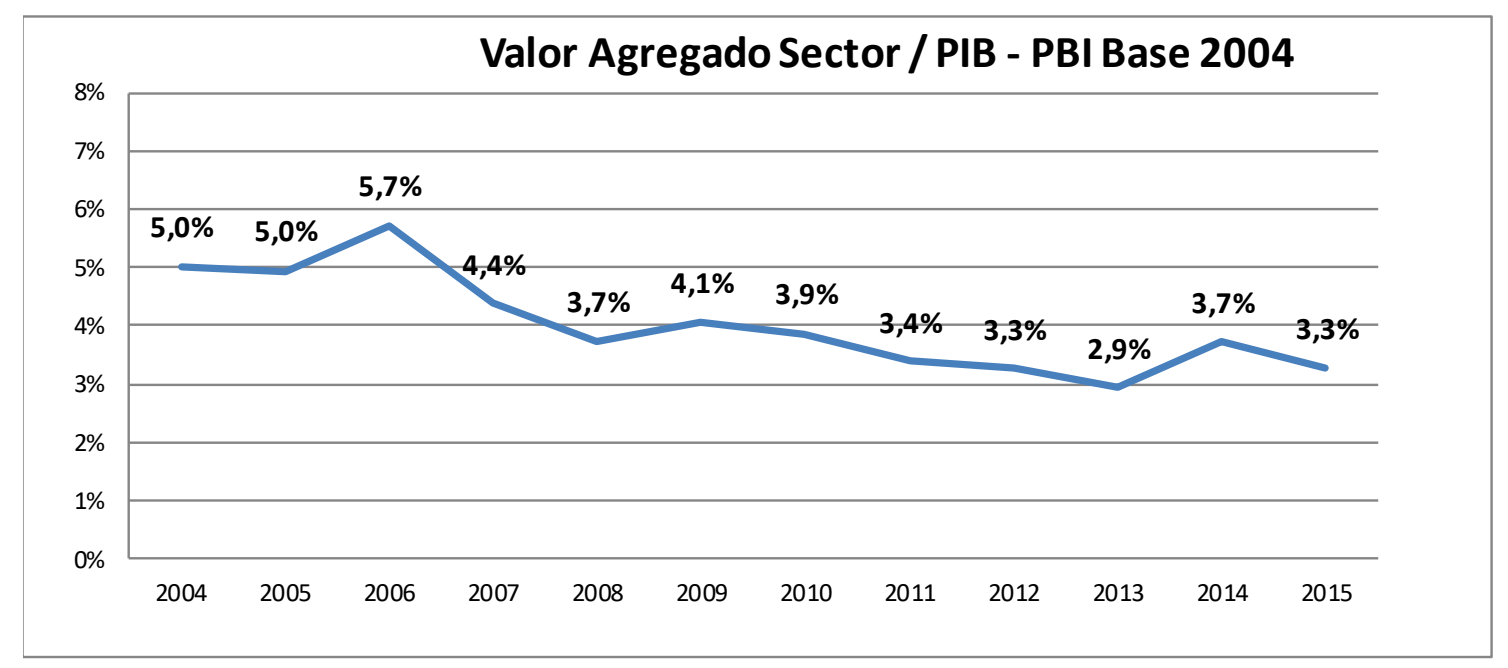

Fuente: INDEC. Sector "Explotación de minas y canteras"

Para entender este proceso, es necesario analizar la dinámica de los precios y niveles de producción del sector, y la evolución del PBI en la Argentina. Así, resulta significativo el aumento de la significatividad de este sector a partir de la crisis económica y social del 2001, en virtud de la fuerte caída del nivel de actividad general, juntamente con la devaluación cambiaria ${ }^{8}$.

Con relación a los precios de los RNR, se registra, tal como se mencionó previamente, un desdoblamiento entre el precio en el mercado doméstico y el mercado externo. El mismo es particularmente importante en el caso del gas natural, lo cual tuvo inevitablemente un efecto negativo sobre el desarrollo de este sector $y$, consecuentemente, en la recaudación fiscal potencial del Estado argentino sobre este sector de la economía.

Al respecto, un instrumento determinante aplicado por el gobierno nacional, además de distintas regulaciones sobre el sector, fue la aplicación de retenciones a la exportación de estos recursos 9 ; en especial, a partir de la vigencia de las retenciones móviles. Para el caso del petróleo crudo, el decreto 310/2002 del ministerio de economía fijó la retención de determinados hidrocarburos en $20 \%$. Luego, la resolución 337/2004 del ministerio de economía elevó la alícuota de determinados productos a $25 \%$; modificada luego por la resolución 532/2004 del ministerio citado. En el año 2007, con niveles de precios internacionales en pleno ascenso, se establece con la resolución 394/2007 del ministerio de economía un sistema de retenciones móviles, que aislaron plenamente el precio doméstico del precio internacional. A partir del 2014, ya con la caída en los precios de los commodities, con la resolución 1077/2014

${ }^{8}$ Los RNR son commodities cuya cotización en dólares está determinada por el mercado internacional; más allá del desdoblamiento de precios mencionado.

9 El gobierno nacional, juntamente con las provincias con RNR, fijaban precios de referencia (por ejemplo, en el caso del gas, se regulaba el precio en boca de pozo según su destino sea residencial, industrial, o de generación de energía). 
se estableció un nuevo esquema que reduce las alícuotas aplicadas (en la actualidad las alícuotas aplicadas rondan el $1 \%$, en un contexto de baja en los precios internacionales $)^{10}$.

En el caso de la minería se aplicó, en particular a partir de 2007, una alícuota entre el $5 \%$ y el $10 \%$, amén de los regímenes de estabilidad fiscal vigentes.

Tal como se visualiza en el siguiente cuadro, la diferencia entre el precio internacional y el precio para el mercado interno del gas natural resultó muy significativa, especialmente entre los años 2003 y $2008^{11}$.

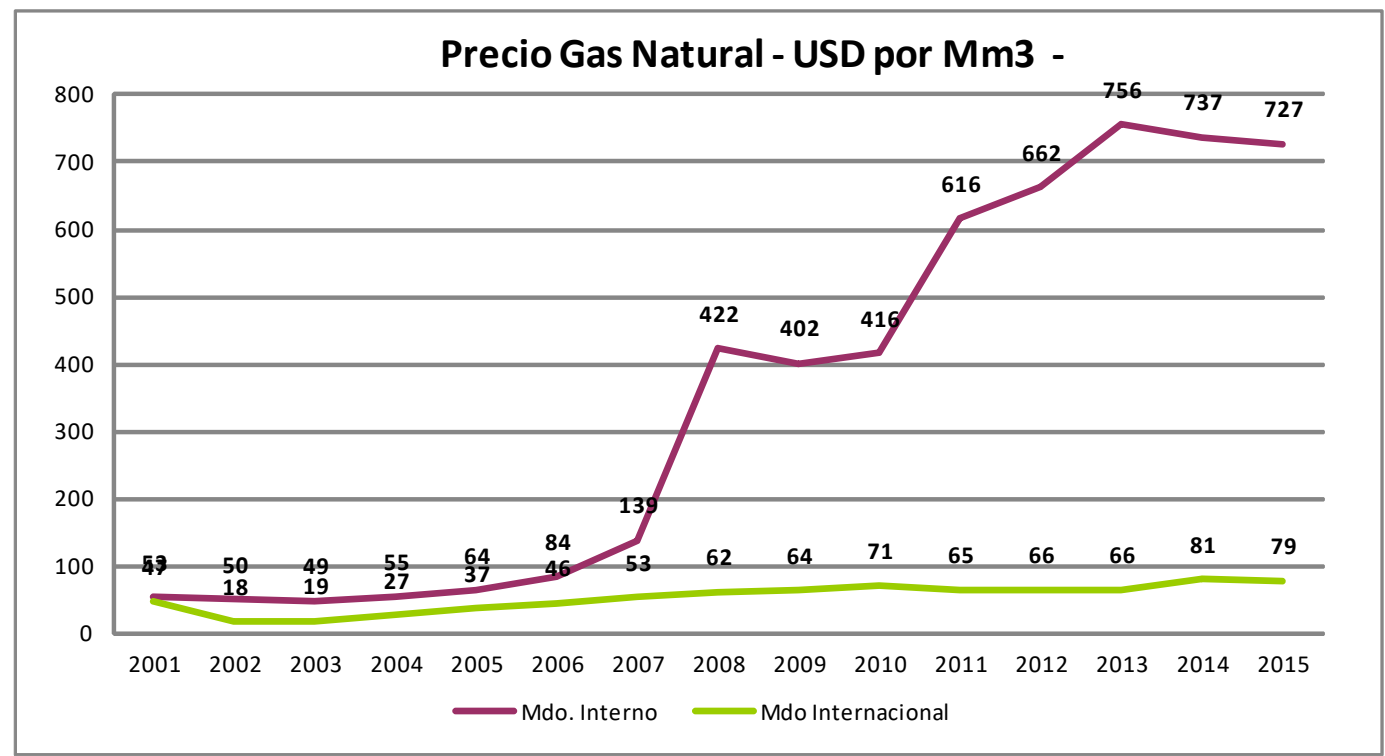

Fuente: Ministerio de Energía

Por su parte, se observa en el caso del petróleo que el descalce de precios entre el mercado interno y externo no resultó tan significativo, excepto en el año 2008.

10 También debe mencionarse que, ante la caída en los niveles de producción, como consecuencia fundamentalmente de las políticas aplicadas, el gobierno instrumentó oportunamente distintos planes para incentivar la producción de gas y petróleo, denominados petróleo plus y gas plus; a partir de los cuales se reconoció un mayor precio a los productores. 11 En el 2015, el precio doméstico resulta incluso superior al internacional, a partir de la caída en este último (al igual que en caso del petróleo crudo). 


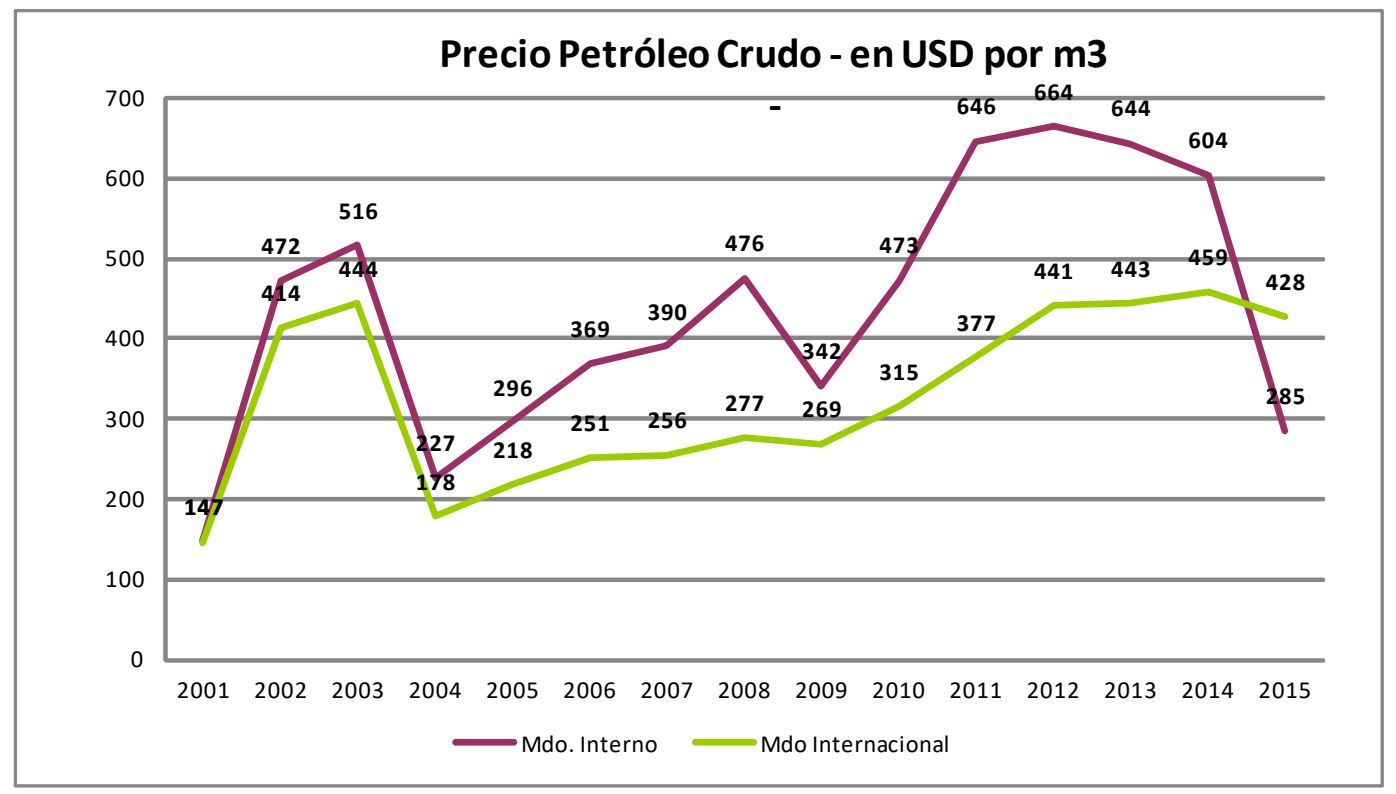

Fuente: Ministerio de Energía

Con relación a los niveles de producción, en el año 2015, la producción de petróleo ascendió a 32 millones de metros cúbicos, mientras que la de gas convencional alcanzó los 43 mil millones de metros cúbicos. Estos guarismos representaron, respectivamente, aproximadamente el $0,8 \%$ y el $1,2 \%$ de la producción mundial. Si se comparan con años anteriores, la producción de petróleo resultó un 35\% menor que en el nivel máximo alcanzado en 1998. Por su parte, la producción de gas resultó un $20 \%$ menor al máximo alcanzado en el año 2004. Del total producido, mientras que hasta el 2006 se exportaba más del 10\% de la producción de gas, desde el 2008 la exportación de gas fue menor al 2\% (o prácticamente nula); (incluso se pasó a importar gas natural en volúmenes que superaron los 10 mil millones de metros cúbicos en 2013, 2014 y 2015); en tanto mientras que en los años 2004 y 2005 se exportaba más del $20 \%$ de la producción de petróleo, en el 2015 se exportó alrededor del $5 \%{ }^{12}$.

\begin{tabular}{|c|c|c|c|c|c|c|c|c|}
\hline \multirow[b]{2}{*}{ Período } & \multicolumn{2}{|c|}{ Producción } & \multicolumn{2}{|c|}{ Reservas } & \multicolumn{2}{|c|}{ Exportación } & \multicolumn{2}{|c|}{ Importación } \\
\hline & \begin{tabular}{|c|} 
Petróleo crudo \\
miles de m3
\end{tabular} & $\begin{array}{l}\text { Gas Natural } \\
\text { mill de } \mathrm{m} 3\end{array}$ & $\begin{array}{c}\text { Petróleo } \\
\text { miles de m3 }\end{array}$ & $\begin{array}{c}\text { Gas } \\
\text { mill de } \mathrm{m} 3\end{array}$ & $\begin{array}{l}\text { Petróleo crudo } \\
\text { miles de m3 }\end{array}$ & $\begin{array}{c}\text { Gas Natural } \\
\text { mill de } \mathrm{m} 3\end{array}$ & \begin{tabular}{|c|} 
Petróleo crudo \\
miles de m3
\end{tabular} & $\begin{array}{l}\text { Gas Natural } \\
\text { mill de } \mathrm{m} 3\end{array}$ \\
\hline 2005 & 38.572 & 51.279 & 349.096 & 438.951 & 8.463 & 6.600 & 262 & 1.734 \\
\hline 2006 & 38.346 & 51.646 & 411.262 & 446.156 & 5.641 & 6.300 & 94 & 1.670 \\
\hline 2007 & 37.905 & 50.971 & 415.913 & 441.974 & 4.046 & 2.662 & 45 & 1.756 \\
\hline 2008 & 37.593 & 50.488 & 400.697 & 398.529 & 3.289 & 1.010 & 6 & 1.448 \\
\hline 2009 & 36.240 & 48.419 & 399.296 & 378.820 & 6.608 & 884 & & 2.672 \\
\hline 2010 & 35.413 & 47.108 & 401.308 & 358.726 & 5.326 & 465 & & 3.612 \\
\hline 2011 & 33.326 & 45.528 & 393.996 & 332.510 & 3.408 & 200 & & 6.909 \\
\hline 2012 & 33.140 & 44.124 & 374.289 & 315.508 & 3.456 & 106 & 247 & 9.506 \\
\hline 2013 & 32.461 & 41.708 & 370.374 & 328.260 & 2.283 & 87 & 421 & 11.809 \\
\hline 2014 & 31.980 & 41.484 & 380.028 & 332.164 & 2.326 & 63 & 548 & 11.899 \\
\hline 2015 & 31.973 & 42.973 & 380.730 & 350.483 & 1.737 & 88 & 292 & 11.388 \\
\hline
\end{tabular}

Fuente: Ministerio de Energía e IAPG

12 Fuente: Instituto Argentino de Petróleo y Gas. 
Con relación a las reservas comprobadas, la Argentina cuenta con un nivel de reservas de gas natural y petróleo, que representan aproximadamente entre 9 y 10 años disponibilidad; y un $0,2 \%$ con relación a las reservas mundiales, Sin embargo, cuenta con un gran potencial en el sector de combustibles no convencionales. Así, las reservas de shale-oil representan el 8,5\% de las reservas mundiales; mientras que las de shale-gas, el $11 \%$ de las reservas mundiales ${ }^{13}$.

Respecto a los recursos mineros (ver mapa territorial en Anexos) en el año 2014, la producción de Argentina de oro ascendió a cerca de $60 \mathrm{mil} \mathrm{kg}$ ( $2 \%$ de la producción mundial); la de cobre a 100 mil $\mathrm{kg}(0,6 \%$ de la producción mundial); y la de plata a casi 1 millón $\mathrm{kg}(3,3 \%$ de la producción mundial).

Como se mencionó previamente, una de las características de la República Argentina consiste en la elevada heterogeneidad regional en la disponibilidad física de los mismos, lo cual no difiere de lo que sucede en otros países. En este sentido, como establece Brosio y Jiménez (2012), una de las características destacables en América Latina, al igual que en el resto del mundo, es la alta concentración geográfica en la producción de estos recursos, lo que crea desbalances horizontales si estos recursos son asignados a los gobiernos subnacionales.

Esto resulta de central importancia teniendo en cuenta, tal como se mencionó previamente, que son las provincias las que detentan el dominio originario de los RNR, de acuerdo con el orden constitucional. La disponibilidad de los RNR en los territorios de las provincias, por tanto, es una de las fuentes de la marcada disparidad económica y fiscal existente entre las mismas.

En el caso del Gas Natural, Neuquén concentra casi la mitad de la producción total. Respecto al Petróleo Crudo, la producción se encuentra más repartida, entre Chubut $(30 \%)$, Santa Cruz $(23 \%)$, Neuquén $(20 \%)$, Mendoza (14\%), Rio Negro $(7 \%)$.

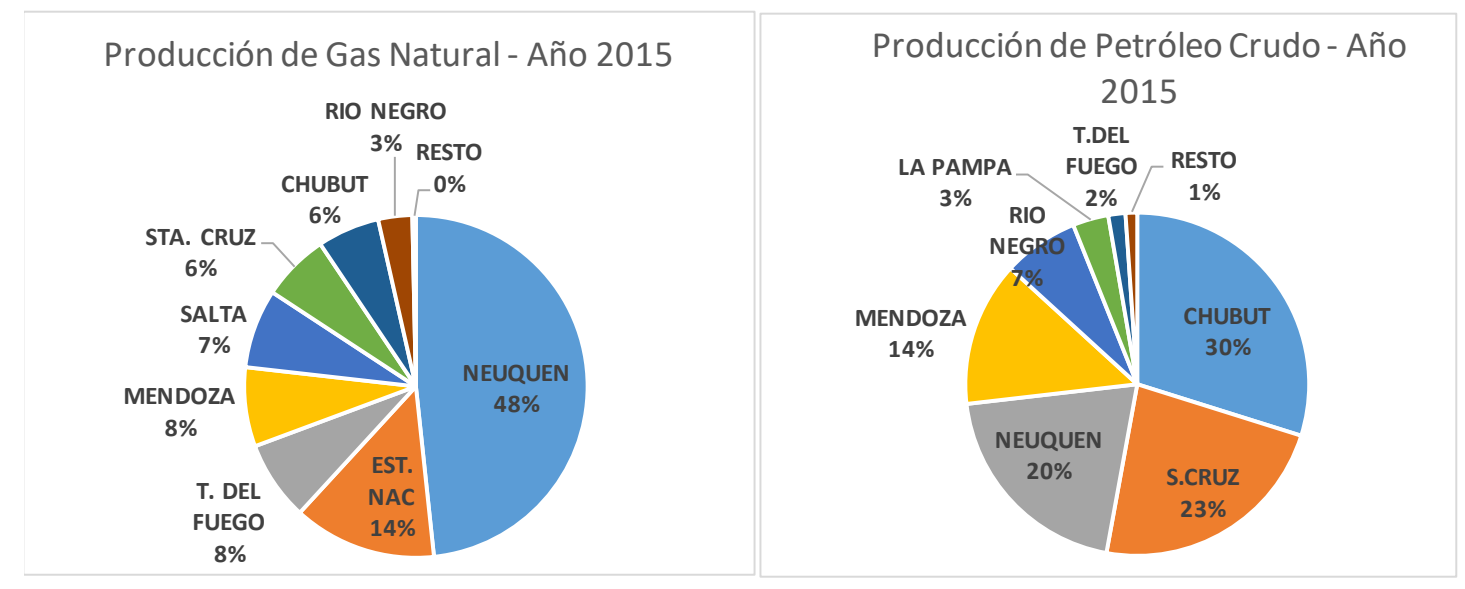

13 Fuente: KPMG: "Estudio Económico sobre Recursos Convencionales, ShaleOil, y Shale Gas en Argentina"; ministerio de hacienda y finanzas de república argentina: "Complejo Petróleo y Gas" y "Minería Metalífera y Rocas de Aplicación". 


\section{Las regalías:}

En nuestro país, la recaudación por regalías, tomando los últimos 22 años, alcanza, en términos acumulados a 31 mil millones de dólares (cerca de USD 24 mil millones recaudados de 2003 a 2015).

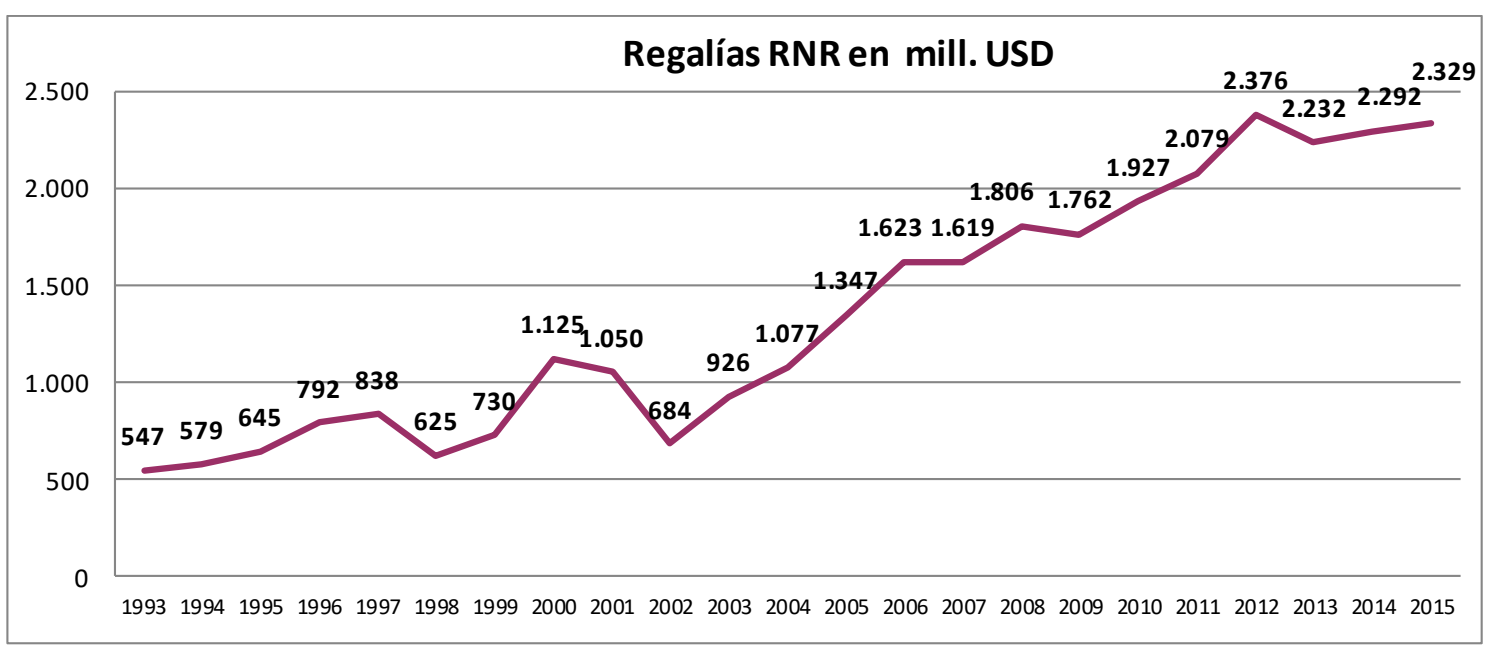

Regalías por Hidrocarburos

\begin{tabular}{|c|c|c|c|c|c|}
\hline \multirow[b]{2}{*}{ Período } & \multicolumn{4}{|c|}{ Producto } & \multirow[b]{2}{*}{$\begin{array}{c}\text { Total } \\
\text { En mill. \$ }\end{array}$} \\
\hline & $\begin{array}{l}\text { Petróleo Crudo } \\
\text { En mill. \$ }\end{array}$ & $\begin{array}{c}\text { Gas Natural } \\
\text { En mill. \$ }\end{array}$ & $\begin{array}{l}\text { Gasolina } \\
\text { En mill. \$ }\end{array}$ & $\begin{array}{c}\text { GLP } \\
\text { En mill. \$ }\end{array}$ & \\
\hline 2004 & $79 \%$ & $16 \%$ & $5 \%$ & $0 \%$ & $100 \%$ \\
\hline 2005 & $78 \%$ & $17 \%$ & $5 \%$ & $0 \%$ & $100 \%$ \\
\hline 2006 & $76 \%$ & $19 \%$ & $5 \%$ & $0 \%$ & $100 \%$ \\
\hline 2007 & $74 \%$ & $20 \%$ & $5 \%$ & $1 \%$ & $100 \%$ \\
\hline 2008 & $71 \%$ & $22 \%$ & $6 \%$ & $1 \%$ & $100 \%$ \\
\hline 2009 & $70 \%$ & $22 \%$ & $6 \%$ & $2 \%$ & $100 \%$ \\
\hline 2010 & $72 \%$ & $21 \%$ & $6 \%$ & $1 \%$ & $100 \%$ \\
\hline 2011 & $73 \%$ & $19 \%$ & $6 \%$ & $2 \%$ & $100 \%$ \\
\hline 2012 & $76 \%$ & $17 \%$ & $7 \%$ & $1 \%$ & $100 \%$ \\
\hline 2013 & $75 \%$ & $17 \%$ & $7 \%$ & $1 \%$ & $100 \%$ \\
\hline 2014 & $76 \%$ & $18 \%$ & $6 \%$ & $0 \%$ & $100 \%$ \\
\hline 2015 & $74 \%$ & $21 \%$ & $5 \%$ & $0 \%$ & $100 \%$ \\
\hline
\end{tabular}

Fuente: En base a Secretaría de Energía

En el cuadro precedente se puede visualizar la significatividad relativa de las regalías por hidrocarburos, según el producto ${ }^{14}$. Se puede ver que las provenientes de petróleo crudo explican tres cuartas partes del total de las mismas.

En los gráficos siguientes, se resume la significatividad de las regalías respecto a los ingresos fiscales de las provincias ${ }^{15}$. En uno de sus puntos máximos (año 2005), las regalías significaron

${ }^{14}$ Las regalías provenientes de hidrocarburos significan aproximadamente el $90 \%$ del total de las mismas.

15 Debe recordarse que en nuestro país los RNR pertenecen a las provincias. 
el $14 \%$ del total de recursos propios del consolidado de 24 provincias. Luego, se observa una tendencia decreciente, hasta alcanzar cerca del $6 \%$ en los últimos años (menos de la mitad que en 2005) $)^{16}$.

La misma situación se registra al medir la importancia de las regalías con relación a los ingresos totales de las provincias. Así, mientras representaron el $6 \%$ en los primeros años de la serie, desde 2013 se encuentran por debajo del $3 \%$ de los ingresos totales de las 24 provincias.

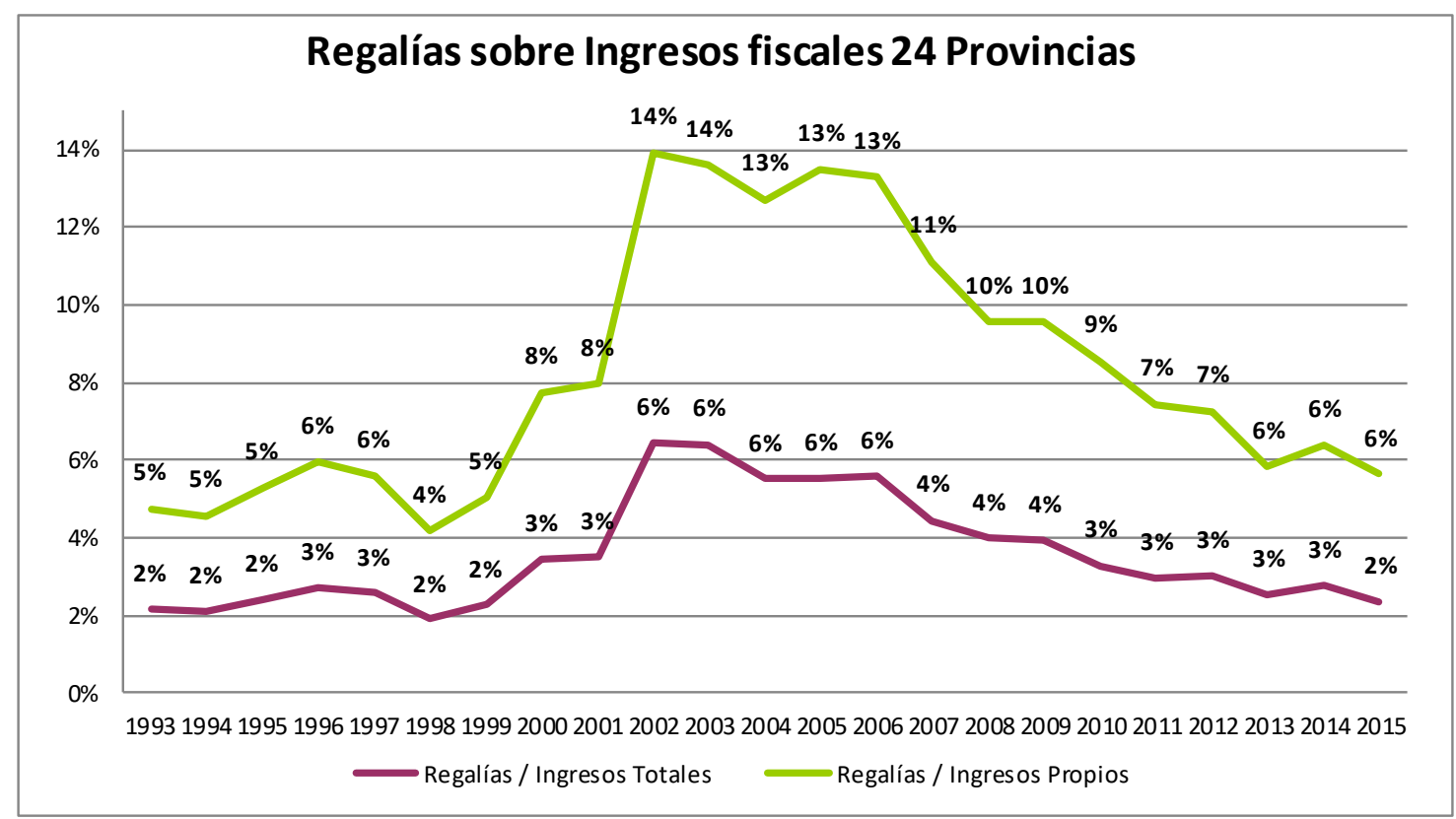

Fuente:DPCFP. Ministerio de Hacienda y Finanzas Públicas

En el cuadro subsiguiente, se resume la estructura de ingresos fiscales de las 24 provincias en años seleccionados. Del mismo, puede observarse, además de la significatividad de las regalías, la evolución de las transferencias automáticas (Ingresos Tributarios de Origen Nacional), de las transferencias presupuestarias provenientes del gobierno nacional (Ingresos por Transferencias Corrientes y de Capital), como así también la dinámica de la recaudación tributaria propia de las provincias (Ingresos Tributarios de Origen Provincial).

${ }^{16} \mathrm{En}$ rigor, los picos máximos se registran en 2002, año en que se produce una significativa caída del PBI. 
Ejecución presupuestaria consolidado 24 provincias. Estructura porcentual

\begin{tabular}{|c|c|c|c|c|c|c|}
\hline & 1993 & 2001 & 2003 & 2008 & 2012 & 2015 \\
\hline I. INGRESOS CORRIENTES & $97 \%$ & $98 \%$ & $98 \%$ & $95 \%$ & $94 \%$ & $94 \%$ \\
\hline Tributarios & $86 \%$ & $84 \%$ & $81 \%$ & $80 \%$ & $81 \%$ & $82 \%$ \\
\hline - De Orígen Provincial & $34 \%$ & $33 \%$ & $34 \%$ & $32 \%$ & $34 \%$ & $35 \%$ \\
\hline - De Orígen Nacional & $52 \%$ & $52 \%$ & $47 \%$ & $48 \%$ & $48 \%$ & $47 \%$ \\
\hline No Tributarios & $8 \%$ & $7 \%$ & $10 \%$ & $7 \%$ & $6 \%$ & $5 \%$ \\
\hline - Regalías & $2 \%$ & $3 \%$ & $6 \%$ & $4 \%$ & $3 \%$ & $2 \%$ \\
\hline - Otros No Tributarios & $6 \%$ & $4 \%$ & $4 \%$ & $3 \%$ & $3 \%$ & $3 \%$ \\
\hline Vta.Bienes y Serv.de la Adm.Publ. & $0 \%$ & $1 \%$ & $1 \%$ & $1 \%$ & $1 \%$ & $1 \%$ \\
\hline Rentas de la Propiedad & $0 \%$ & $1 \%$ & $1 \%$ & $1 \%$ & $0 \%$ & $0 \%$ \\
\hline Transferencias Corrientes & $2 \%$ & $4 \%$ & $5 \%$ & $6 \%$ & $6 \%$ & $6 \%$ \\
\hline IV. INGRESOS DE CAPITAL & $3 \%$ & $2 \%$ & $2 \%$ & $5 \%$ & $6 \%$ & $6 \%$ \\
\hline Recursos Propios de Capital & $0 \%$ & $0 \%$ & $0 \%$ & $0 \%$ & $0 \%$ & $0 \%$ \\
\hline Transferencias de Capital & $1 \%$ & $0 \%$ & $0 \%$ & $4 \%$ & $5 \%$ & $6 \%$ \\
\hline Dismin. de la Inversión Financiera & $2 \%$ & $2 \%$ & $2 \%$ & $1 \%$ & $1 \%$ & $0 \%$ \\
\hline VI. INGRESOS TOTALES (I+IV) & $100 \%$ & $100 \%$ & $100 \%$ & $100 \%$ & $100 \%$ & $100 \%$ \\
\hline
\end{tabular}

Fuente: en base a DPCFP. Ministerio de Hacienda y Finanzas Públicas

Se destaca, por ejemplo, un aumento en la participación de los ingresos por transferencias discrecionales (presupuestarias), en contraste con una disminución relativa de las transferencias automáticas. Por su parte, los ingresos tributarios propios no registran una modificación relevante en el financiamiento de las provincias.

Si se analiza la relación entre las regalías y el valor agregado del sector económico, puede obtenerse una medida de la "alícuota implícita" vigente. Como se desprende del segundo gráfico (PBI Base 2004), la relación mencionada oscila cerca de un promedio del 12\%, en línea con el alícuota legal promedio registrada en las provincias; y en torno al $0,5 \%$ respecto al PBI.

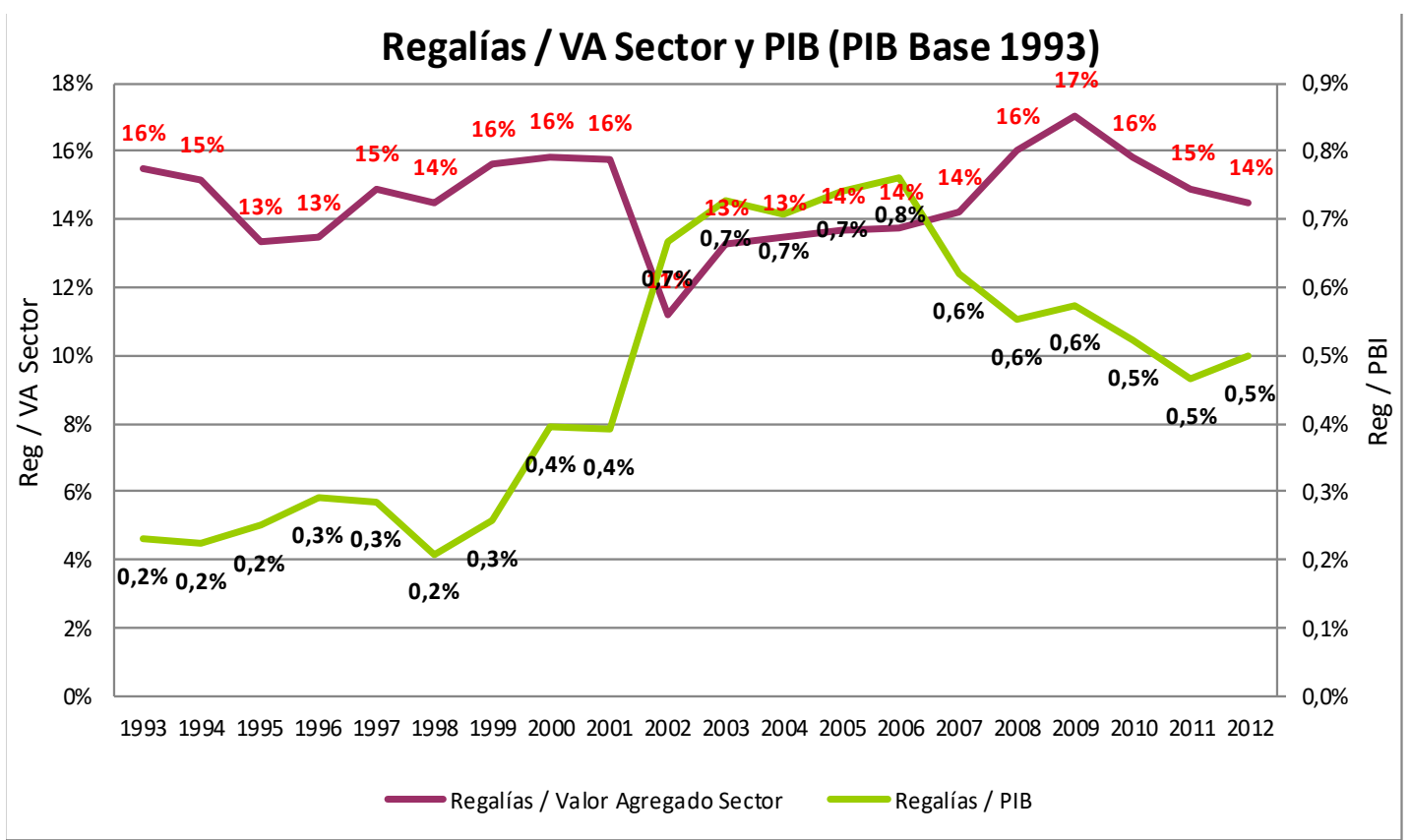

Fuente:DPCFP y SSPE. Ministerio de Hacienda y Finanzas Públicas. 


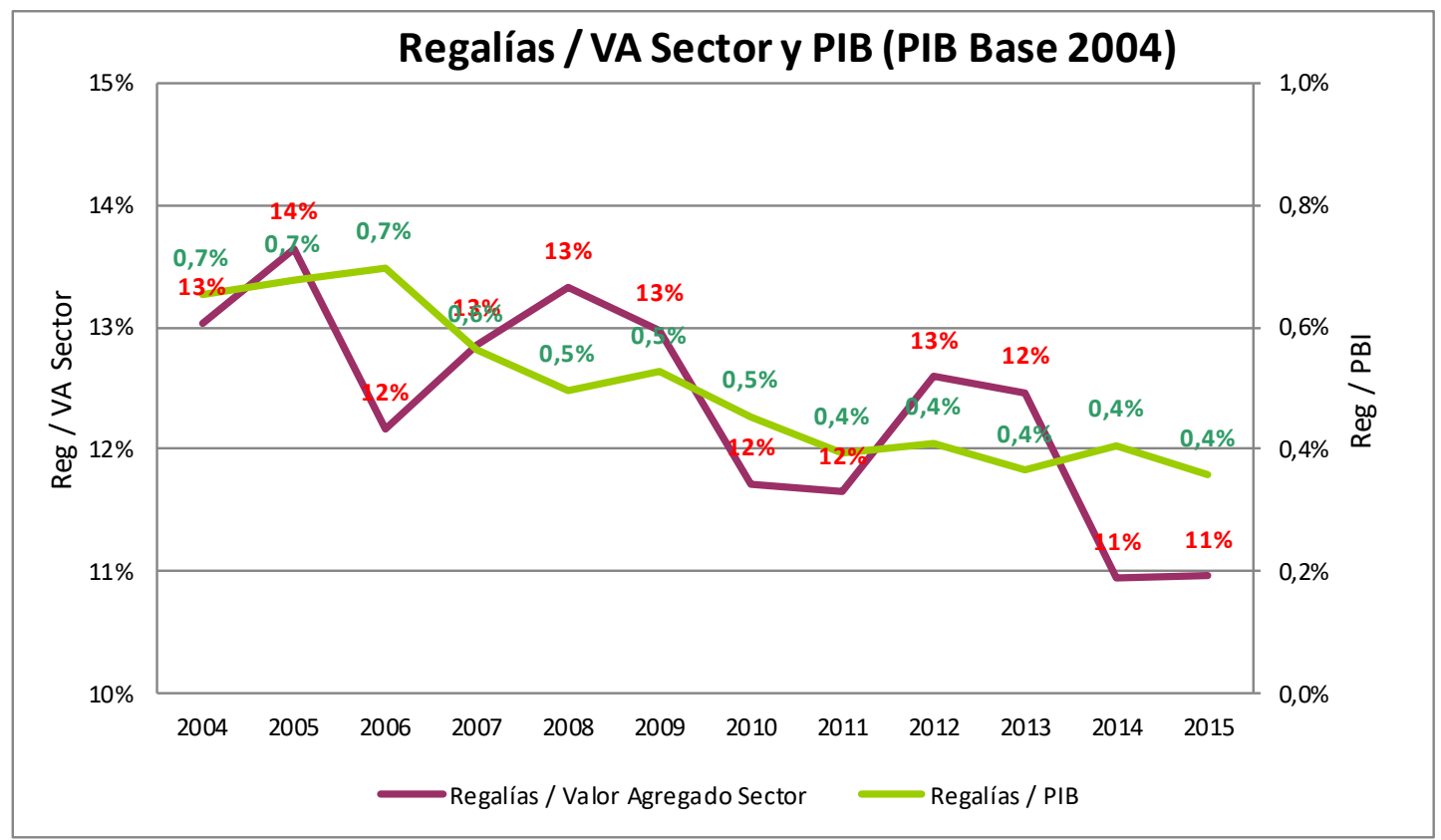

Fuente:DPCFP y SSPE. Ministerio de Hacienda y Finanzas Públicas.

Como consecuencia lógica de la focalización de la producción en ciertas provincias, y la propiedad de la misma por parte de los gobiernos subnacionales, la distribución de las regalías se encuentra tambien geográficamente concentrada.

En este sentido, si bien el análisis se realiza sobre las 24 provincias, casi del $90 \%$ del ingreso por regalías (año 2015) está concentrado en cinco provincias, fundamentalmente provincias patagónicas ricas en hidrocarburos. Así, Neuquén concentra el $26 \%$ de la recaudación total de regalías de este país; Chubut el $18 \%$; Santa Cruz el $18 \%$; Mendoza el $12 \%$ y Rio Negro el $15 \%$ del total ${ }^{17}$.

17 Debe destacarse que estas provincias se caracterizan, además, por tener una baja densidad poblacional (amplio territorio, con relativa baja cantidad de habitantes); lo que determina a su vez que, a partir del sistema de coparticipación federal de impuestos, reciban los mayores ingresos por habitante en concepto de transferencias desde el gobierno nacional. 


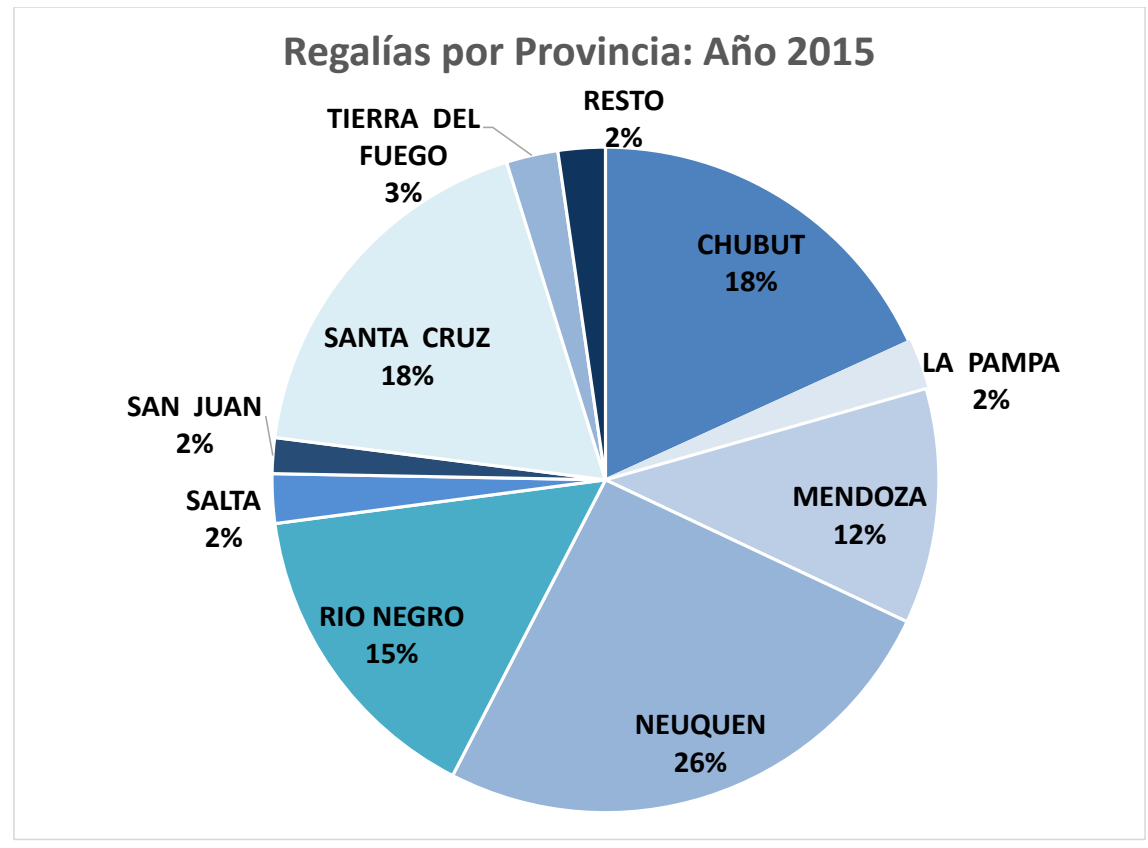

Dentro de la estructura de ingresos fiscales de cada una de estas provincias, las regalías significan una parte sustancial. Como se observa en la tabla, en el período 2004 a 2007, se registraron valores máximos para la mayoría de las provincias. En efecto las regalías explicaron el $56 \%$ de los recursos propios de Santa Cruz, y el $30 \%$ de sus ingresos totales; en Chubut, las mismas explicaron el $57 \%$ de los recursos propios, y el $37 \%$ de sus ingresos totales; mientras que, en Neuquén, significaron, en dicho período, el $60 \%$ de los recursos propios, y el $46 \%$ de sus ingresos totales.

\begin{tabular}{|c|c|c|c|c|c|c|c|c|}
\hline \multirow[b]{2}{*}{ Provincia } & \multicolumn{4}{|c|}{ Regalías/Ingresos Propios } & \multicolumn{4}{|c|}{ Regalías /Ingresos Totales } \\
\hline & $\begin{array}{c}\text { Media } \\
20042007\end{array}$ & $\begin{array}{c}\text { Media } \\
20082010\end{array}$ & $\begin{array}{c}\text { Media } \\
20112014\end{array}$ & 2015 & $\begin{array}{c}\text { Media } \\
20042007\end{array}$ & $\begin{array}{c}\text { Media } \\
20082010\end{array}$ & $\begin{array}{c}\text { Media } \\
2011\end{array}$ & 2015 \\
\hline RIO NEGRO & $34 \%$ & $29 \%$ & $29 \%$ & $39 \%$ & $13 \%$ & $10 \%$ & $10 \%$ & $16 \%$ \\
\hline SANTA CRUZ & $56 \%$ & $32 \%$ & $33 \%$ & $34 \%$ & $30 \%$ & $17 \%$ & $19 \%$ & $19 \%$ \\
\hline CHUBUT & $57 \%$ & $42 \%$ & $38 \%$ & $27 \%$ & $37 \%$ & $28 \%$ & $25 \%$ & $17 \%$ \\
\hline NEUQUEN & $60 \%$ & $39 \%$ & $31 \%$ & $24 \%$ & $46 \%$ & $29 \%$ & $21 \%$ & $17 \%$ \\
\hline MENDOZA & $31 \%$ & $24 \%$ & $16 \%$ & $14 \%$ & $15 \%$ & $11 \%$ & $7 \%$ & $6 \%$ \\
\hline SALTA & $8 \%$ & $22 \%$ & $12 \%$ & $9 \%$ & $2 \%$ & $6 \%$ & $3 \%$ & $2 \%$ \\
\hline LA PAMPA & $10 \%$ & $9 \%$ & $10 \%$ & $8 \%$ & $4 \%$ & $3 \%$ & $4 \%$ & $4 \%$ \\
\hline TIERRA DEL FUEGO & $30 \%$ & $22 \%$ & $14 \%$ & $8 \%$ & $15 \%$ & $11 \%$ & $7 \%$ & $4 \%$ \\
\hline SAN JUAN & $2 \%$ & $12 \%$ & $10 \%$ & $6 \%$ & $0 \%$ & $2 \%$ & $3 \%$ & $2 \%$ \\
\hline CATAMARCA & $25 \%$ & $13 \%$ & $10 \%$ & $4 \%$ & $6 \%$ & $3 \%$ & $2 \%$ & $1 \%$ \\
\hline FORMOSA & $7 \%$ & $2 \%$ & $2 \%$ & $1 \%$ & $1 \%$ & $0 \%$ & $0 \%$ & $0 \%$ \\
\hline CORRIENTES & $3 \%$ & $2 \%$ & $2 \%$ & $1 \%$ & $1 \%$ & $0 \%$ & $1 \%$ & $0 \%$ \\
\hline ENTRE RIOS & $6 \%$ & $7 \%$ & $5 \%$ & $1 \%$ & $2 \%$ & $2 \%$ & $2 \%$ & $0 \%$ \\
\hline MISIONES & $4 \%$ & $6 \%$ & $2 \%$ & $1 \%$ & $1 \%$ & $1 \%$ & $1 \%$ & $0 \%$ \\
\hline JUJUY & $1 \%$ & $1 \%$ & $1 \%$ & $0 \%$ & $0 \%$ & $0 \%$ & $0 \%$ & $0 \%$ \\
\hline SANTIAGO DEL ESTERO & $0 \%$ & $0 \%$ & $0 \%$ & $0 \%$ & $0 \%$ & $0 \%$ & $0 \%$ & $0 \%$ \\
\hline CABA & $0 \%$ & $0 \%$ & $0 \%$ & $0 \%$ & $0 \%$ & $0 \%$ & $0 \%$ & $0 \%$ \\
\hline BUENOS AIRES & $0 \%$ & $0 \%$ & $0 \%$ & $0 \%$ & $0 \%$ & $0 \%$ & $0 \%$ & $0 \%$ \\
\hline CORDOBA & $0 \%$ & $0 \%$ & $0 \%$ & $0 \%$ & $0 \%$ & $0 \%$ & $0 \%$ & $0 \%$ \\
\hline CHACO & $0 \%$ & $0 \%$ & $0 \%$ & $0 \%$ & $0 \%$ & $0 \%$ & $0 \%$ & $0 \%$ \\
\hline LA RIOJA & $0 \%$ & $0 \%$ & $0 \%$ & $0 \%$ & $0 \%$ & $0 \%$ & $0 \%$ & $0 \%$ \\
\hline SAN LUIS & $0 \%$ & $0 \%$ & $0 \%$ & $0 \%$ & $0 \%$ & $0 \%$ & $0 \%$ & $0 \%$ \\
\hline SANTA FE & $0 \%$ & $0 \%$ & $0 \%$ & $0 \%$ & $0 \%$ & $0 \%$ & $0 \%$ & $0 \%$ \\
\hline TUCUMAN & $0 \%$ & $0 \%$ & $0 \%$ & $0 \%$ & $0 \%$ & $0 \%$ & $0 \%$ & $0 \%$ \\
\hline
\end{tabular}

Fuente:DPCFP. Ministerio de Hacienda y Finanzas Públicas 
Al comparar la significatividad de las regalías respecto a los ingresos propios en los distintos períodos, se registra una caída en la significatividad de las mismas entre el primer período y el 2015 (por ejemplo, en Neuquén de 60\% en el primer período a 24\% en 2015); aunque algunas como Rio Negro y San Juan aumentan su significatividad en el último período. Sin embargo, aún tienen una relevancia importante dentro de la estructura de ingresos de algunas provincias; fundamentalmente de las más ricas en estos recursos.

\section{Modelo Econométrico}

Para poder testear las hipótesis planteadas en el apartado correspondiente (las regalías y el comportamiento fiscal de las provincias), en esta sección se explora, a través de un modelo econométrico, la relación funcional que existe entre los ingresos fiscales por regalías sobre los RNR con respecto al gasto total, el esfuerzo fiscal, y la planta de personal (cantidad de empleados públicos provinciales) de las 23 provincias más CABA. Es decir, que impacto tiene los ingresos fiscales provenientes de RNR sobre aquellas variables fiscales fundamentales de los gobiernos subnacionales.

Para una correcta especificación, se parte de un modelo que incluye como variables explicativas, además de las regalías, aquellas variables que puedan determinar el comportamiento de las variables a explicar. De esta forma, se postulan las siguientes ecuaciones a ser estimadas:

\footnotetext{
$\mathrm{Gtpc}_{\text {it }}=\mathrm{a}+\mathrm{b}_{\text {regpc }}$ it $+\mathrm{c}$ tapc $_{\text {it }}+\mathrm{dtdpc}_{\text {it }}+$ pbgpc $_{\text {it }}+\mathrm{gtpc}_{\text {it }-1}+u_{i}+e_{\text {it }}$$$
\text { ippc }_{\text {it }}=a+b_{\text {regpc }} \text { it }+c_{\text {tapc }} \text { it }+d \text { tdpc }_{\text {it }}+\text { pbgpc }_{\text {it }}+\text { ippc }_{\text {it }-1}+u_{i}+e_{b} \text { it }
$$

Personal $=a+b$ regpc $_{i t}+c$ tapc $i t+d$ tdpc $i t+p_{i t}$ bgpc $_{i t}+$ personal $_{\text {it }-1}+u_{i}+e_{c i t}$
}

Donde: gtpc $=$ gasto total per cápita; regpc=regalías per cápita; tapc $=$ transferencias automáticas per cápita; $t \mathrm{dpc}=$ transferencias discrecionales per cápita; pbgpc= producto bruto geográfico per cápita; ippc= ingresos tributarios propios de las provincias per cápita; personal= cantidad de empleados públicos (en niveles absolutos) ${ }^{18}$. Las variables gtpc-1; ippc-1; y personal-1 son las variables explicadas correspondientes al período anterior (variables rezagadas). Por último, el termino e corresponde al error en cada ecuación; y el término u corresponde al efecto fijo.

18 La información relativa a la planta de personal (cantidad de empleados públicos provinciales) se desprende de la publicación de la DPCFP, Ministerio de Hacienda y Finanzas Públicas. La misma corresponde al total de empleados provinciales (en planta y contratados) correspondientes a Administración Central, Organismos Descentralizados y Cuentas Especiales. 
A partir de estas ecuaciones, se utilizan datos en panel de 24 provincias, abarcando el período 1993 a 2015, y se estima un modelo de efectos fijos ${ }^{19}$. Las variables están en moneda constante $^{20}$, y definidas en función de la cantidad de habitantes de cada jurisdicción (a excepción de personal). Asimismo, en los modelos se incluye la variable dependiente rezagada como variable. La introducción de la variable explicada rezagada, como variable explicativa, busca captar el efecto inercial que puede registrarse en el comportamiento de esta variable (ver Ajit Karnik, Mala Lalvani (2005)).

Los estudios relacionados con el gasto público total (siempre definidas per cápita) tienden a analizar la existencia del denominado flypaper effect ${ }^{21}$. En los modelos relacionados con los ingresos tributarios propios como variable explicada, se puede analizar el denominado "esfuerzo fiscal" (medido en este trabajo a partir de los ingresos tributarios propios de las jurisdicciones $)^{22}$. Por su parte, el análisis de la planta de personal, en función de las variables explicativas, puede ayudar a evaluar las hipótesis relacionadas con el concepto de "Estado Rentista" citado previamente.

Con relación a las variables explicativas, las transferencias del gobierno nacional se pueden clasificar en dos categorias: las transferencias automáticas, que están constituidas por el régimen de coparticipación federal y regímenes especiales, que, por definición, no dependen de las decisiones presupuestarias de las autoridades fiscales nacionales. Por su parte, las transferencias discrecionales, constituyen aquellas enmarcadas en programas presupuestarios del gobierno nacional destinados a transferir recursos a las provincias, y que efectivamente dependen de las decisiones presupuestarias de las autoridades fiscales.

Respecto al gasto total per cápita como variable explicada, sería de esperar un efecto positivo y significativo sobre aquel en las tres variables explicativas; entendiendo fundamentalmente que estas variables no resultan de la presión tributaria de las jurisdicciones sobre sus ciudadanos ( $y$ el costo político que ello conlleva), sino que provienen o bien de transferencias

19 Datos en panel: cuando se dispone de datos en panel, en este caso, observaciones de distintas provincias en varios períodos de tiempo, son utilizados modelos de efectos fijos (o modelos de efectos aleatorios). Para discernir sobre cual utilizar se realiza el test de Hausman (ver Sosa Escudero, 1999).

${ }^{20}$ En base a índices de www. Inflaciónverdadera.com

${ }^{21} \mathrm{Fly}$ paper effect: ante incrementos en las transferencias se genera una expansión del gasto público subnacional mayor que si se hubiese experimentado un aumento equivalente (en la misma magnitud del incremento en las transferencias) del ingreso "propio" en la jurisdicción local. El punto básico, derivado del enfoque positivo de la escuela del "public choice" tiene que ver con los efectos provocados por la separación entre las decisiones de gasto y su financiamiento.

${ }^{22}$ La definición de Esfuerzo fiscal (EF) resulta compleja. Bird y Smat (2002), establecen que la manera más adecuada de definirlo es como la relación entre la recaudación efectivamente ingresada y la recaudación potencial, definida esta última en base a un estándar de capacidad fiscal y de tasa impositiva (ver Porto, 2006). En la práctica, diversos autores, intentan medir el EF como el cociente entre Ingresos Tributarios Propios de cada jurisdicción (en Argentina, fundamentalmente Ingresos Brutos, Inmobiliario, Sellos, entre otros). 
nacionales, o bien de la disponibilidad o no de RNR. Lo mismo cabría esperar cuando la variable explicada es la planta de personal de las provincias.

Por su parte, podría esperarse un efecto negativo de las variables explicativas sobre el esfuerzo fiscal. En este sentido, los gobiernos subnacionales podrían "utilizar" estos ingresos para no explotar adecuadamente sus bases de recaudación propia, en virtud del costo político involucrado. Desde otra óptica, podría utilizar estos ingresos para reducir la presión tributaria (política de "empobrecer al vecino") aspectos comentados previamente en el documento.

Respecto al gasto total per cápita, tal como se expone en la siguiente tabla, se observan coeficientes positivos y significativos en las variables explicativas con relación al gasto total. En particular, se registra un coeficiente de 0,76 en las transferencias automáticas; de 0,56 en las transferencias discrecionales; de 0,3 en regalías; de 0,02 en el producto bruto geográfico; y de 0,57 en la variable dependiente rezagada. Estos valores abalan las siguientes conclusiones:

El alto valor de los coeficientes de las transferencias (automáticas y discrecionales) con relación al coeficiente correspondiente al producto bruto geográfico sugiere un efecto "flypaper"23. En efecto el coeficiente de las transferencias $(0,76$ y 0,56 , tapc y tdpc respectivamente) resulta considerablemente mayor al correspondiente al producto bruto geográfico (cercano a cero).

Variable explicada: Gasto Total Per Cápita
Estimación Efectos Fijos
\begin{tabular}{|lc|}
\hline \multicolumn{2}{|c|}{ Gasto Per Cápita } \\
\hline regpc & $0.307^{* * *}$ \\
& {$[0.098]$} \\
tapc & $0.767^{* * *}$ \\
& {$[0.102]$} \\
tdpc & $0.562^{* * *}$ \\
& {$[0.183]$} \\
pbgpc & $0.021^{* * *}$ \\
& {$[0.007]$} \\
gtpc_1 & $0.572^{* * *}$ \\
& {$[0.047]$} \\
Constant & $-224.244^{* *}$ \\
& {$[96.183]$} \\
Observations & 528 \\
R-squared & 0.926 \\
Number of prov & 24 \\
Robust standard errors in brackets \\
$* * *$ p $<0.01,{ }^{* *} p<0.05,{ }^{*} p<0.1$ \\
\end{tabular}

23 En efecto el producto bruto geográfico resulta una variable proxi del comportamiento de la recaudación tributaria propia de las provincias. 
En el caso de las transferencias discrecionales, resultan significativas, aunque con un coeficiente menor en valor absoluto en comparación con las transferencias automáticas. Como menciona Artana (2012), el menor valor de los coeficientes puede estar explicado en el hecho de que un aumento en las transferencias discrecionales es considerado como una mejora o "ingreso transitorio", las cuales no son utilizadas para aumentar el nivel del gasto de la misma manera que si fueran un aumento en el "ingreso permanente" asociado a las transferencias automáticas.

En el caso de las regalías, puede observarse que las mismas resultan significativas respecto al gasto total, aunque con un coeficiente menor que las transferencias (incluso discrecionales). En este sentido, la alta incertidumbre que rodea a las mismas puede llevar a considerar sus aumentos (disminución) como una mejora (desmejora) en los ingresos transitorios, con implicancias similares a las descriptas para las transferencias discrecionales. También, debe tenerse en cuenta otras consideraciones. En primer lugar, las provincias con RNR suelen utilizar ingresos por regalías como garantía para la obtención de endeudamiento; por lo cual estos ingresos son utilizados en algunos casos, al menos parcialmente para la amortización de deudas $^{24}$. En segundo lugar, puede existir una interacción con las decisiones de política económica del gobierno nacional. Así, al producirse un ciclo alcista en los ingresos por regalías, los gobiernos subnacionales pueden esperar que la Nación neutralice parcialmente tal aumento, ya sea a través de retenciones, política tarifaria, política tributaria, presupuestaria, etc.; lo cual desista a los gobiernos propietarios de estos recursos a aumentar sus erogaciones $^{25}$.

Asimismo, el coeficiente de la variable rezagada (positivo y significativo), marca un efecto inercial en el comportamiento de esta variable. En otros términos, el gasto total per cápita de los gobiernos provinciales se ve afectado por el comportamiento de dicha variable en el período anterior.

Con respecto a los ingresos tributarios propios per cápita, a excepción de las transferencias automáticas y la variable dependiente rezagada, las variables explicativas del modelo no resultan significativas. En el caso de las transferencias automáticas, tienen un efecto incluso positivo sobre la variable explicada (coeficiente positivo 0,068). Estos resultados resultan consistentes con la hipótesis de existencia del fly paper effect ${ }^{26}$.

\footnotetext{
${ }^{24}$ La amortización de deudas no se incluye en el cómputo del gasto de las provincias. Resultan gastos "debajo de la línea".

25 En este sentido Piffano (2014) consigna que el gobierno nacional, particularmente en los últimos años del período analizado, descentralizo el "ajuste fiscal" en los gobiernos subnacionales; tanto al avanzar sobre la tributación de impuestos directos como a través de las retenciones; a la vez que desalentó la actividad del sector de RNR, al punto de convertir al país en importador neto de estos recursos.

${ }^{26}$ Los mayores ingresos por regalías y transferencias no son usados para reducir la presión tributaria, lo cual es un efecto previsto desde la óptica del flypaper effect. Al explicar la
} 
Por último, resulta significativa la variable dependiente rezagada, siempre per cápita, (ingresos tributarios propios del ejercicio anterior) con un coeficiente positivo 0,9 , lo cual denota un marcado efecto inercial.

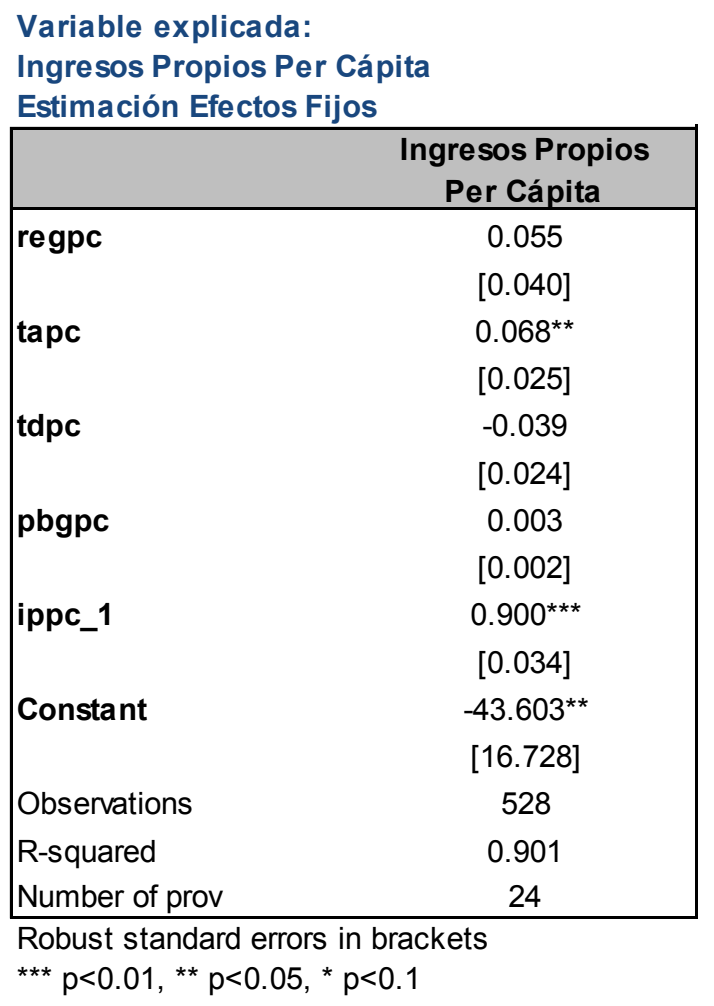

Respecto a la planta de personal, al igual que en el caso de los ingresos tributarios propios, resultan significativos y con signo positivo la variable dependiente rezagada (sugiriendo una marcada inercia en el comportamiento de esta variable); y las transferencias automáticas (coeficiente positivo 0,002). Así, mayores transferencias automáticas incidirían en un aumento en la contratación de empleados provinciales, ceteris paribus. Por el contrario, las regalías y las transferencias discrecionales resultan no significativas.

existencia del FlyPaper Effect y la ausencia de una reducción en el esfuerzo fiscal (en efecto aumenta la presión fiscal), Porto (2001) refiere a los hallazgos de Bird y Fiszbein (mimeo, 1996) con referencia a Colombia: "On the whole, the evidence to date in Colombia in any case appears to support more the so called "flypaper effect"...than the "fiscal effort" hypothesis that increased transfers result in reduced local taxes". Respecto al coeficiente positivo de las transferencias automáticas sobre los ingresos tributarios propios, podría explicarse en el efecto expansivo de aquellas transferencias sobre la demanda agregada de la provincia (y así sobre la base recaudatoria). 
Variable explicada: Planta de Personal

Estimación Efectos Fijos

\begin{tabular}{|lc|}
\hline & Personal \\
\hline regpc & -0.001 \\
& {$[0.001]$} \\
tapc & $0.002^{* * *}$ \\
& {$[0.001]$} \\
& 0.000 \\
pbgpc & {$[0.001]$} \\
& 0.000 \\
per_1 & {$[0.000]$} \\
& $0.998^{* * *}$ \\
Constant & {$[0.003]$} \\
& -1.019 \\
Observations & {$[0.670]$} \\
R-squared & 528 \\
Number of prov & 0.980 \\
Robust standard & 24 \\
\hline
\end{tabular}

Robust standard errors in brackets

${ }^{* * *} p<0.01,{ }^{* *} p<0.05,{ }^{*} p<0.1$

\section{Comentarios finales}

Del trabajo presentado se pueden destacar las siguientes consideraciones:

- La abundancia de Recursos no Renovables (RNR), específicamente hidrocarburos y minería; su potencial exploratorio, y los elevados precios de los últimos años, determinan su papel preponderante en la economía de los países de Latino América. La República Argentina está caracterizada por la abundancia de estos recursos, concentrada, espacialmente los hidrocarburos, en las provincias patagónicas.

- El carácter de recurso no renovable, juntamente con las características intrínsecas del sector, tienen implicancias económicas y fiscales distintivas. En este sentido, por ejemplo, si el gobierno limita su explotación en determinado año, ello no impide que al año siguiente se lo haga, sin perder, en términos Inter-temporales, los niveles de producción.

- Con relación los RNR y el federalismo fiscal, la mayoría de los argumentos favorecen la centralización de los RNR en el gobierno central; en tanto los argumentos en favor de la descentralización son de carácter fundamentalmente histórico, político e institucional, tal como sucede en la Argentina.

- En virtud del dominio de las provincias sobre los recursos no renovables, la motivación de este trabajo consiste en analizar el efecto que tiene la disponibilidad de los RNR en el comportamiento fiscal de las provincias; en particular en la década del 2000. De esta manera, partiendo de los lineamientos conceptuales provenientes de la escuela del "public choice" se pretende determinar si la disponibilidad de RNR ha determinado una 
reducción o "relajamiento" en el "Esfuerzo Fiscal" de las provincias a la hora de recaudar el resto de los tributos subnacionales; y/o un incentivo a sobredimensionar el gasto y la cantidad de empleados públicos de las mismas.

- Del estudio econométrico, respecto al gasto total per cápita, se registra un efecto significativo (y positivo) de las transferencias (en mayor medida las automáticas), sobre el nivel del gasto; y aporta evidencia a la existencia del efecto flypaper. Las regalías resultan significativas (y signo positivo), con un valor del coeficiente menor.

- En el caso de las regalías, lo mismo que las transferencias discrecionales, la alta incertidumbre que las rodea, puede llevar a considerar sus aumentos (disminuciones) como mejoras (desmejoras) como ingresos transitorios; lo cual produciría un efecto menor sobre el nivel del gasto (respecto a si los aumentos fueran permanentes). Por otra parte, en el caso de las regalías, el gobierno nacional regula y, en los últimos años de la serie en particular, ha limitado la explotación subnacional de los RNR, reduciendo los niveles de producción; pasando la Argentina de ser un país exportador a uno importador de estos recursos.

- Respecto al esfuerzo fiscal, resulta significativa y con coeficiente positivo las transferencias automáticas. En tanto, las regalías y las transferencias discrecionales no resultan significativas. Así, tanto los ingresos por regalías como por las transferencias desde el gobierno nacional no conllevarían a una reducción en los ingresos tributarios propios (en el caso de las transferencias automáticas tiene un efecto incluso positivo).

- En el caso de la planta de personal, resultan significativas y con coeficiente positivo las transferencias automáticas (las cuales serían utilizadas para contratación de más empleados provinciales). En tanto, las regalías y las transferencias discrecionales resultan no significativas.

- Resulta significativa, en todas las especificaciones planteadas, la variable dependiente rezagada (incluida como variable explicativa), lo que denota un efecto inercial en el comportamiento de las variables explicadas. 


\section{Bibliografía}

AJIT KARNIK, MALA LALVANI (2005): Urban Local Governments and the Flypaper Effect: Evidence from Maharashtra, India. University of Mumbai.

ALTOMONTE, H. y SANCEZ, R (2016); "Hacia una Nueve Gobernanza de los Recursos Naturales en América Latina y Caribe"; CEPAL. Santiago, Chile.

ALTOMONTE, H. (2013);" Recursos Naturales en UNASUR: "Situación y Tendencias para una agenda de desarrollo Regional"; CEPAL-UNASUR. Santiago, Chile.

ARTANA D. y TEMPLADO I. (2011); "Is the Argentine Revenue Effort "Too" High?" En Alberto Porto y Fernando Navajas (editores de contenido) "Progresos en Economía del Sector Público". Ediciones EDICON. Buenos Aires.

ARTANA D., AUGUSTE S., CRISTINI M., MOSKOVITZ C. y TEMPLADO I. (2012); "SubNational Revenue Mobilization in Latin American and Caribean Countries: The Case of Argentina". Fundación de Investigaciones Latinoamericanas, Banco Interamericano de Desarrollo (IDB). WorkingPaper Series No IDB-WP-297.

BIRD R. SMART, M. (2002); "Intergovernmental Fiscal Transfers: International Lessons for Developing Countries".

BROSIO, G y JIMENEZ, J.P. (2012); "Intergobernmental allocation of revenue from natural resources: finding a balance between centripetal and centrifugal pressure". En Brosio, G. y JIMENEZ, J.P. (eds.); "Descentralization and reform in LatinAmerica", Edward Elgar Publishing paper, pp.290-313. 
EINSTOSS TINTO, ALEJANDRO (2016); "Análisis de la evolución reciente de la renta petrolera en Argentina. CECE.

FMI (2012); "Fiscal regimes for extractive industries: design and implementation"; Fiscal Affairs Deparment."

FRETES CIBILS V. (2016);" Descentralizando los ingresos fiscales en América Latina. Porqué y Cómo". Libro "Prioridades, problemas y oportunidades de las reformas federales". EDICON.

GERVASONI, C. (2010); "A rantiertheory of subnationalregimes"; Princeton University.

GERVASONI, C. (2016); "Es vaca muerta una maldición. Consecuencias de las rentas petroleras para el federalismo, la economía y la democracia". Libro "Prioridades, problemas y oportunidades de las reformas federales". EDICON.

GOMEZ SABAINI, J.C., JIMENEZ, J.P.; MORAN J.P. (2015); "El impacto fiscal de la explotación de recursos no renovables en los países de América Latina y el Caribe"; CEPAL.

KPMG (2014); "Estudio Económico sobre Recursos Convencionales, Shale Oil, y Shale Gas en Argentina".

MELAMUD, A. y ROZENWURCEL, G. (2016); "Tributación de recursos naturales en América del Sur"; ASAP.

MINISTERIO DE HACIENDA Y FINANZAS DE REPÚBLICA ARGENTINA (2011); "Complejo Petróleo y Gas"; Serie: Producción Regional por Complejos Productivos".

MINISTERIO DE HACIENDA Y FINANZAS DE REPÚBLICA ARGENTINA (2016); "Minería Metalifera y Rocas de Aplicación"; Serie: Informes de Cadenas de Valor".

OATES W. (1972); "Fiscal Federalim"; Ed. Instituto de Estudios de Administración Local, Madrid (1977).

PIFFANO, H. (2005); Nota 1: "Una revisión de la literatura sobre asignación de potestades fiscales".

PIFFANO, H. (2005); Nota 10: "Los recursos no renovables en un sistema federal de gobierno"; UNLP.

PIFFANO, H. (2014); "Opciones Tributarias de los Gobiernos Subnacionales en el Actual Escenario Federal". SAP.

PORTO A. (2001); "Modelos de Federalismo Fiscal"; UNLP. 
PORTO, A. (2004); "Disparidades regionales y federalismo fiscal"; UNLP.

PORTO, A. (2006); "Capacidad, Necesidad, esfuerzo fiscal y distribución secundaria de la coparticipación"; UNLP.

SOSA ESCUDERO, W. (1999); “Tópicos de Econometría Aplicada". UNLP.

TANZI V. (2016);" Rethinking Fiscal Federalism. A Critical Evaluation". Libro "Prioridades, problemas y oportunidades de las reformas federales". EDICON.

Anexos 
Mapa. Extracción de hidrocarburos por cuencas sedimentarias. Año 2015
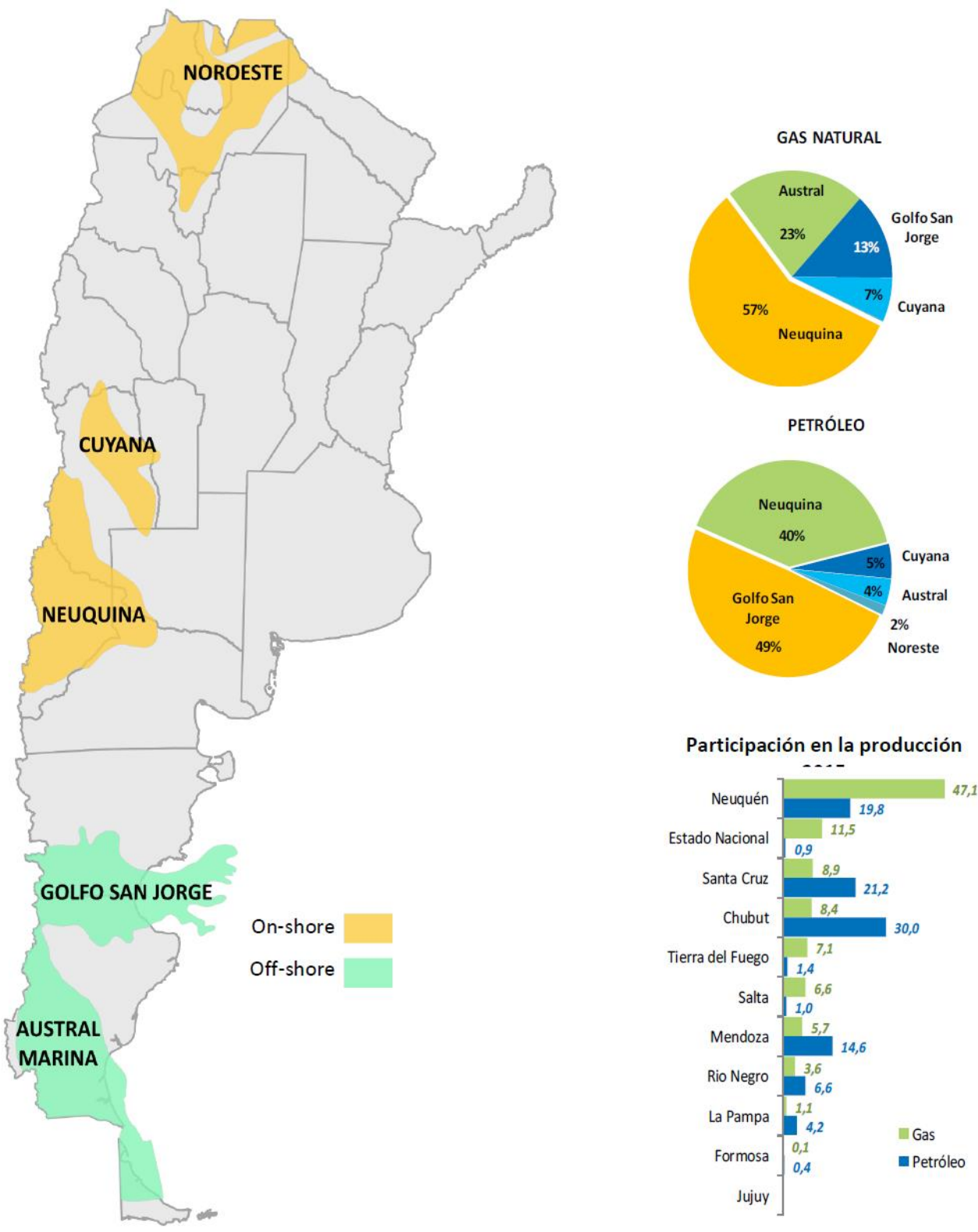

Fuente: elaboración propia con base en datos de MEyM. 


\section{Distribución territorial de la Cadena Minera Metalífera}

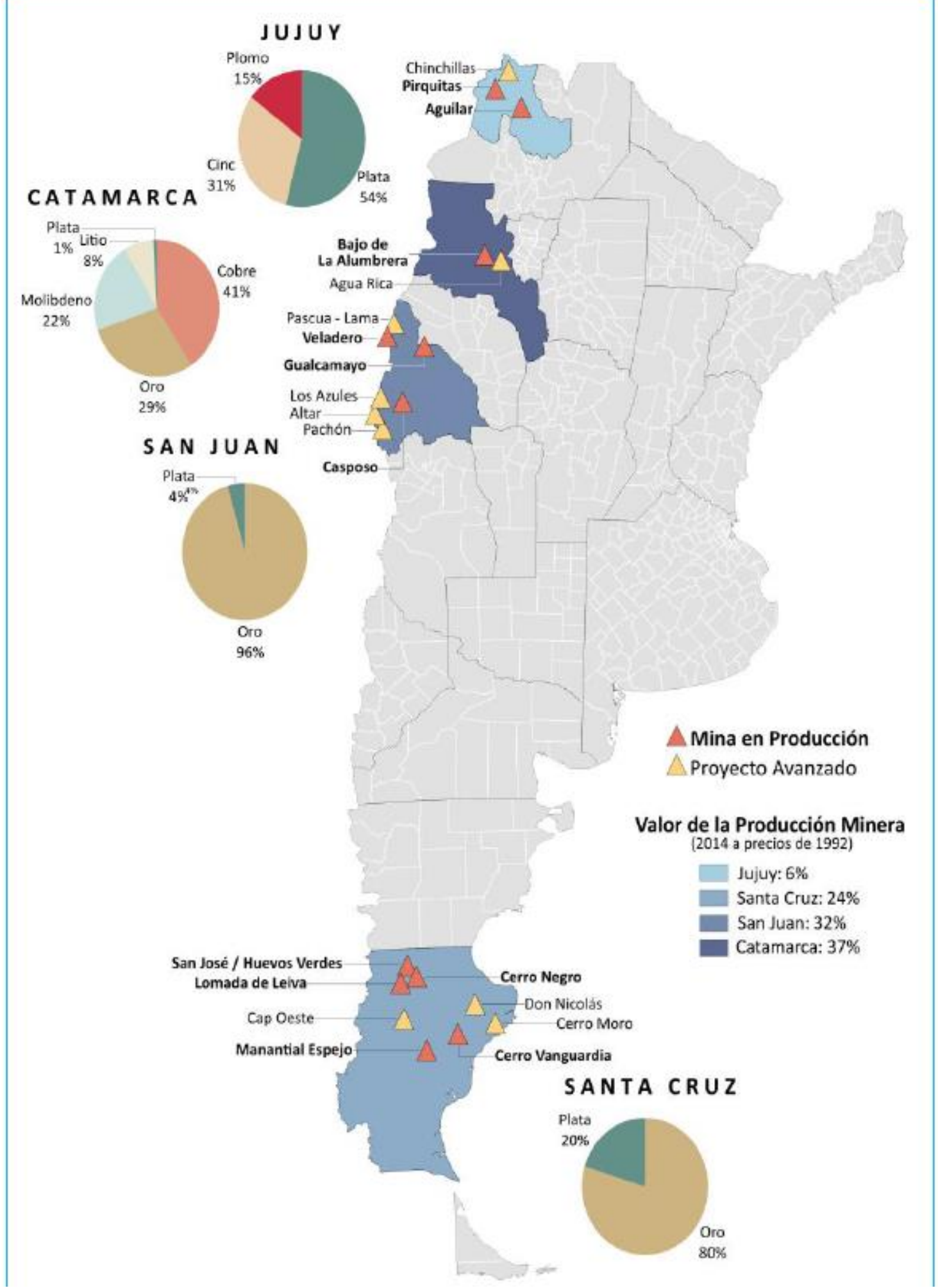

